\title{
EMBEDDINGS FOR SPACES OF LORENTZ-SOBOLEV TYPE
}

\author{
ANDREAS SEEGER AND WALTER TREBELS
}

\begin{abstract}
The purpose of this paper is to characterize all embeddings for versions of Besov and Triebel-Lizorkin spaces where the underlying Lebesgue space metric is replaced by a Lorentz space metric. We include two appendices, one on the relation between classes of endpoint MikhlinHörmander type Fourier multipliers, and one on the constant in the triangle inequality for the spaces $L^{p, r}$ when $p<1$.
\end{abstract}

\section{INTRODUCTION}

We consider Lorentz space variants of the classical function space scales of Sobolev, Besov and Triebel-Lizorkin spaces for distributions on $\mathbb{R}^{d}$. We use the traditional Fourier analytical definition ( $c f$. [29]) and work with an inhomogeneous Littlewood-Paley decomposition $\left\{\Lambda_{k}\right\}_{k=0}^{\infty}$ which is defined as follows. Pick a $C^{\infty}$ function $\beta_{0}$ such that $\beta_{0}(\xi)=1$ for $|\xi| \leq 3 / 2$ and $\beta_{0}(\xi)=0$ for $|\xi| \geq 7 / 4$. For $k \geq 1$ let $\beta_{k}(\xi)=\beta_{0}\left(2^{-k} \xi\right)-\beta_{0}\left(2^{1-k} \xi\right)$. Define $\Lambda_{k}$ via the Fourier transform by $\widehat{\Lambda_{k} f}=\beta_{k} \widehat{f}, k=0,1,2, \ldots$.

Let $\mathcal{Y}$ be a rearrangement invariant quasi-Banach space of functions on $\mathbb{R}^{d}$, and define

$$
\begin{aligned}
\|f\|_{B_{q}^{s}[\mathcal{Y}]} & =\left(\sum_{k} 2^{k s q}\left\|\Lambda_{k} f\right\|_{\mathcal{Y}}^{q}\right)^{1 / q}, \\
\|f\|_{F_{q}^{s}[\mathcal{Y}]} & =\left\|\left(\sum_{k}\left|2^{k s} \Lambda_{k} f\right|^{q}\right)^{1 / q}\right\|_{\mathcal{Y}} .
\end{aligned}
$$

When the functors $B_{q}^{s}$ and $F_{q}^{s}$ are applied to the Lebesgue spaces $\mathcal{Y}=L^{p}$ one gets the usual classes of Besov spaces $B_{q}^{s}\left[L^{p}\right] \equiv B_{p, q}^{s}$ and Triebel-Lizorkin spaces $F_{q}^{s}\left[L^{p}\right] \equiv F_{p, q}^{s}$. Here we take for $\mathcal{Y}$ a Lorentz space $L^{p, r}$, see 92 for definitions and a review of basic facts. Of course $L^{p, p}=L^{p}$. It is also customary to write $B_{q}^{s}\left[L^{p, r}\right]=B_{(p, r), q}^{s}, F_{q}^{s}\left[L^{p, r}\right]=F_{(p, r), q}^{s}$, although for better readability we prefer the functorial notation. For $q=2$ one obtains the Lorentz versions of the Hardy-Sobolev spaces, also denoted by $H_{(p, r)}^{s}$.

2010 Mathematics Subject Classification. 46E35, 46E30, 42B15, 42B25.

Key words and phrases. Lorentz spaces, Besov spaces, Sobolev spaces, Triebel-Lizorkin spaces, Embeddings, Sobolev embeddings, Hörmander multiplier theorem.

Research supported in part by National Science Foundation grant DMS 1500162. 
For the range $1<p<\infty$ the space $H_{(p, r)}^{s} \equiv F_{2}^{s}\left[L^{p, r}\right]$ can be identified with a variant of Bessel-potential spaces ( $c f .[23$, ch.V]), namely we have

$$
\|f\|_{F_{2}^{s}\left[L^{p, r}\right]} \approx\left\|(I-\Delta)^{s / 2} f\right\|_{p, r}, \quad 1<p<\infty .
$$

These spaces have been used repeatedly in the literature (see e.g. [24], [5], [15], 9]), although our original motivation came from a result about embeddings in [8]. Applications suggest natural questions about the relation between these spaces, in particular the relation between Besov and LorentzSobolev spaces. Our two main theorems characterize all embeddings which involve one space in the $B_{q}^{s}\left[L^{p, r}\right]$ family and one space in the $F_{q}^{s}\left[L^{p, r}\right]$ family.

Theorem 1.1. Let $s_{0}, s_{1} \in \mathbb{R}, 0<p_{0}, p_{1}<\infty, 0<q_{0}, q_{1}, r_{0}, r_{1} \leq \infty$. The embedding

$$
B_{q_{0}}^{s_{0}}\left[L^{p_{0}, r_{0}}\right] \hookrightarrow F_{q_{1}}^{s_{1}}\left[L^{p_{1}, r_{1}}\right]
$$

holds if and only if one of the following six conditions is satisfied.

(i) $s_{0}-s_{1}>d / p_{0}-d / p_{1}>0$.

(ii) $s_{0}>s_{1}, p_{0}=p_{1}, r_{0} \leq r_{1}$.

(iii) $s_{0}-s_{1}=d / p_{0}-d / p_{1}>0, q_{0} \leq r_{1}$.

(iv) $s_{0}=s_{1}, p_{0}=p_{1} \neq q_{1}, r_{0} \leq r_{1}, q_{0} \leq \min \left\{p_{1}, q_{1}, r_{1}\right\}$.

(v) $s_{0}=s_{1}, p_{0}=p_{1}=q_{1} \geq r_{0}, r_{0} \leq r_{1}, q_{0} \leq \min \left\{p_{1}, r_{1}\right\}$.

(vi) $s_{0}=s_{1}, p_{0}=p_{1}=q_{1}<r_{0}, r_{0} \leq r_{1}, q_{0}<p_{1}$.

Theorem 1.2. Let $s_{0}, s_{1} \in \mathbb{R}, 0<p_{0}, p_{1}<\infty, 0<q_{0}, q_{1}, r_{0}, r_{1} \leq \infty$. The embedding

$$
F_{q_{0}}^{s_{0}}\left[L^{p_{0}, r_{0}}\right] \hookrightarrow B_{q_{1}}^{s_{1}}\left[L^{p_{1}, r_{1}}\right]
$$

holds if and only if one of the following six conditions is satisfied.

(i) $s_{0}-s_{1}>d / p_{0}-d / p_{1}>0$.

(ii) $s_{0}>s_{1}, p_{0}=p_{1}, r_{0} \leq r_{1}$.

(iii) $s_{0}-s_{1}=d / p_{0}-d / p_{1}>0, r_{0} \leq q_{1}$.

(iv) $s_{0}=s_{1}, p_{0}=p_{1} \neq q_{0}, r_{0} \leq r_{1}, q_{1} \geq \max \left\{p_{0}, q_{0}, r_{0}\right\}$.

(v) $s_{0}=s_{1}, p_{0}=p_{1}=q_{0} \leq r_{1}, r_{0} \leq r_{1}, q_{1} \geq \max \left\{p_{0}, r_{0}\right\}$

(vi) $s_{0}=s_{1}, p_{0}=p_{1}=q_{0}>r_{1}, r_{0} \leq r_{1}, q_{1}>p_{0}$.

Remark 1.3. The interesting cases deal with the critical relation

$$
s_{0}-s_{1}=d / p_{0}-d / p_{1},
$$

when $p_{0}<p_{1}$, and when $p_{0}=p_{1}$. The case $p_{0}<p_{1}$ in (iii) of the two theorems sheds some light on the sharp embedding theorems by Jawerth [13 and Franke [7. Part (iii) of Theorem 1.2 extends and improves Jawerth's theorem stating that $F_{q_{0}}^{s_{0}}\left[L^{p_{0}}\right] \hookrightarrow B_{p_{0}}^{s_{1}}\left[L^{p_{1}}\right]$ for any $q_{0} \leq \infty$, under the assumption (6) $), p_{0}<p_{1}$. Part (iii) of Theorem 1.1 extends the dual version of Franke stating that $B_{p_{1}}^{s_{0}}\left[L^{p_{0}}\right] \hookrightarrow F_{q_{1}}^{s_{1}}\left[L^{p_{1}}\right]$, for any $q_{1}>0$, again under the assumption (6),$p_{0}<p_{1}$. For the Hardy-Sobolev case, $q_{1}=2$, a 
partial result of Theorem 1.1, (iii) can be found in [8, under the additional assumption $r_{0} \leq r_{1}$.

Remark 1.4. We shall see in Appendix $\mathrm{A}$ that an application of parts (iii) of Theorems 1.1 and 1.2 in tandem is useful to compare sharp versions of the Hörmander multiplier theorem in [18] and [9].

Parts (iv), (v), (vi) of both theorems deal with the endpoint case $s_{0}=s_{1}$, $p_{0}=p_{1}$ in (6). The conditions on the $q_{i}$ and $r_{i}$ are now more restrictive. The sufficiency of the conditions in (iv), (v), (vi) for (4), (5), resp., follow from corresponding embedding results for the spaces $\ell^{q}\left(L^{p, r}\right)$ and $L^{p, r}\left(\ell^{q}\right)$ for sequences of functions $f=\left\{f_{k}\right\}_{k=0}^{\infty}$. It turns out that these results can be reduced to two types of triangle inequalities for Lorentz spaces. We note that the two strict inequalities in parts (vi) of both theorems can be traced to the failure of a triangle inequality in $L^{1, \rho}$ for $\rho>1$. While considering the results in parts (iv), (v) of the two theorems we came across the question on how the constants in a generalized triangle inequality for quasi-norms in $L^{p, \rho}$ depend on $\rho$ when $p<1$ and $p<\rho<\infty$. This dependence is not crucial to our results but may be interesting in its own right, and we include a result as Appendix B.

The above theorems are complemented by more straightforward results about embeddings within the $B_{q}^{s}\left[L^{p, r}\right]$ and $F_{q}^{s}\left[L^{p, r}\right]$ scales of spaces.

Theorem 1.5. Let $s_{0}, s_{1} \in \mathbb{R}, 0<p_{0}, p_{1}<\infty, 0<q_{0}, q_{1}, r_{0}, r_{1} \leq \infty$. The embedding

$$
B_{q_{0}}^{s_{0}}\left[L^{p_{0}, r_{0}}\right] \hookrightarrow B_{q_{1}}^{s_{1}}\left[L^{p_{1}, r_{1}}\right]
$$

holds if and only if one of the following four conditions is satisfied.

(i) $s_{0}-s_{1}>d / p_{0}-d / p_{1}>0$.

(ii) $s_{0}>s_{1}, p_{0}=p_{1}, r_{0} \leq r_{1}$.

(iii) $s_{0}-s_{1}=d / p_{0}-d / p_{1}>0, q_{0} \leq q_{1}$.

(iv) $s_{0}=s_{1}, p_{0}=p_{1}, r_{0} \leq r_{1}, q_{0} \leq q_{1}$.

Theorem 1.6. Let $s_{0}, s_{1} \in \mathbb{R}, 0<p_{0}, p_{1}<\infty, 0<q_{0}, q_{1}, r_{0}, r_{1} \leq \infty$. The embedding

$$
F_{q_{0}}^{s_{0}}\left[L^{p_{0}, r_{0}}\right] \hookrightarrow F_{q_{1}}^{s_{1}}\left[L^{p_{1}, r_{1}}\right]
$$

holds if and only if one of the following four conditions is satisfied.

(i) $s_{0}-s_{1}>d / p_{0}-d / p_{1}>0$.

(ii) $s_{0}>s_{1}, p_{0}=p_{1}, r_{0} \leq r_{1}$.

(iii) $s_{0}-s_{1}=d / p_{0}-d / p_{1}>0, r_{0} \leq r_{1}$.

(iv) $s_{0}=s_{1}, p_{0}=p_{1}, r_{0} \leq r_{1}, q_{0} \leq q_{1}$.

It is noteworthy that in statements (iii) of Theorem 1.5, for the critical relation (6) and $p_{0}<p_{1}$ the parameters $r_{0}, r_{1}$ in the Besov-Lorentz embeddings can be chosen arbitrary. Likewise in Theorem[1.6, (iii), the parameters 
$q_{0}, q_{1}$ are arbitrary. This result can be quickly derived from Theorems 1.1 and 1.2 (see $\$ 6$ ) and extends results by Jawerth [13] for the Lebesgue space cases $p_{0}=r_{0}, p_{1}=r_{1}$.

This paper. In 92 we shall review basic facts on Lorentz spaces and related spaces $\ell^{q}\left(L^{p, r}\right)$ and $L^{p, r}\left(\ell^{q}\right)$ for sequences of functions $f=\left\{f_{k}\right\}_{k=0}^{\infty}$. In $\lceil 3$ we also discuss various examples demonstrating the sharpness of the results; see in particular the overview in 3.1 for a guide where to find the proof of each necessary condition. In $\$ 4$ we prove embedding relations between $\ell^{q}\left(L^{p, r}\right)$ and $L^{p, r}\left(\ell^{q}\right)$, for fixed $p, r$, which imply the sufficiency of the conditions in parts (iv)-(vi) of Theorems 1.1 and 1.2. In $\$ 5$ we give the proofs of the Lorentz improvements of the embedding theorems by Franke and Jawerth (i.e. parts (iii) of Theorems 1.1 and 1.2). The proofs of sufficiency are concluded in 86 .

We have formulated our results for the homogeneous spaces $B_{q}^{s}\left(L^{p, r}\right)$, $F_{q}^{s}\left(L^{p, r}\right)$, but the proofs can be extended to cover homogeneous versions $\dot{B}_{q}^{s}\left(L^{p, r}\right)$ and $\dot{F}_{q}^{s}\left(L^{p, r}\right)$, defined via the usual homogeneous dyadic frequency decompositions, (cf. [31]). The corresponding results for the homogeneous variants then hold for the critical scale invariant embeddings.

In Appendix $\mathrm{A}$ we discuss some classes of Fourier multipliers and state some open problems. In Appendix $\mathrm{B}$ we prove the above mentioned result on the constant for the triangle inequality for $L^{p, r}$ when $p<1, r<\infty$.

Acknowledgement. We thank the referee of this paper for a careful reading and for pointing out possible further directions: First, the spaces considered here fit in the axiomatic approach by Hedberg and Netrusov [10]. Secondly, it might be of interest to also replace the sequence spaces in our definitions by their Lorentz versions and settle analogous embedding questions.

\section{REVIEW OF BASIC FACTS ON LORENTZ SPACES}

We review some basic facts about Lorentz spaces, and refer the reader to [3], 4], 12], [26] for more information.

2.1. Lorentz spaces via the distribution function. Let $(X, \mu)$ be a measure space. For a measurable function $f$ we let

$$
\mu_{f}(\alpha)=\mu(\{x \in X:|f(x)|>\alpha\}),
$$

be the distribution function and $f^{*}(t)=\inf \left\{\alpha: \mu_{f}(\alpha) \leq t\right\}$ be the nonincreasing rearrangement of $|f|$. We shall assume that $\mu$ is non-atomic (i.e. every set of positive measure has a subset of smaller positive measure).

For $0<p, r<\infty$, the standard quasi-norm on the Lorentz space $L^{p, r}$ is given by

$$
\|f\|_{p, r}=\left(\frac{r}{p} \int\left[t^{1 / p} f^{*}(t)\right]^{r} \frac{d t}{t}\right)^{1 / r}
$$


moreover $\|f\|_{p, \infty}=\sup _{t} t^{1 / p} f^{*}(t), 0<p<\infty$. There is also an alternative description via the distribution function, namely

$$
\|f\|_{p, r}=\left(r \int\left[\mu_{f}(\alpha)^{1 / p} \alpha\right]^{r} \frac{d \alpha}{\alpha}\right)^{1 / r}
$$

and $\|f\|_{p, \infty}=\sup _{\lambda} \lambda \mu_{f}(\lambda)^{1 / p}$. One checks this for simple functions first, and then applies the monotone convergence theorem. The analogue for the case $r=\infty$ is done in Stein-Weiss [26, p.191], and the case $r<\infty$, for simple functions relies on similar summation by parts arguments.

For later use we state the usual embedding for fixed $p$, namely $L^{p, r} \hookrightarrow L^{p, q}$ for $r \leq q$. In fact there is the sharp inequality

$$
\|f\|_{p, q} \leq\|f\|_{p, r}, \quad 0<r \leq q \leq \infty .
$$

A proof using rearrangements is in Stein-Weiss [26, p.192], but the proof of (11) could also be based on (10), $c f$. Lemma B.2 in the appendix.

2.2. Sequences of functions. The study of function spaces crucially relies on the study of the sequence spaces $L^{p, r}\left(\ell^{q}\right)$ and $\ell^{q}\left(L^{p, r}\right)$. We shall work with the quasi-norms

$$
\begin{aligned}
\|f\|_{L^{p, r}\left(\ell^{q}\right)} & :=\left\|\left(\sum_{k}\left|f_{k}\right|^{q}\right)^{1 / q}\right\|_{p, r}, \\
\|f\|_{\ell^{q}\left(L^{p, r}\right)} & :=\left(\sum_{k}\left\|f_{k}\right\|_{p, r}^{q}\right)^{1 / q} .
\end{aligned}
$$

Throughout the paper the domains of the sequences will usually be $\mathbb{N} \cup\{0\}$, or a subset of it, but it could be any finite or countable set with counting measure.

2.3. Powers. It will be convenient to use formulas for the distribution and rearrangement functions of $|f|^{\sigma}$, for any $\sigma>0$, namely

$$
\mu_{|f|^{\sigma}}(\alpha)=\mu_{f}\left(\alpha^{1 / \sigma}\right)
$$

and

$$
\left(|f|^{\sigma}\right)^{*}(t)=\left(f^{*}(t)\right)^{\sigma} .
$$

These follow directly from the definition of distribution and rearrangement functions. An immediate consequence is

$$
\left\||f|^{\sigma}\right\|_{L^{p / \sigma, r / \sigma}}=\|f\|_{L^{p, r}}^{\sigma} .
$$

Moreover, for sequences of functions $f=\left\{f_{k}\right\}$,

$$
\begin{aligned}
& \left\|\left\{\left|f_{k}\right|^{\sigma}\right\}\right\|_{L^{p / \sigma, r / \sigma\left(\ell^{q / \sigma}\right)}}=\|f\|_{L^{p, r}\left(\ell^{q}\right)}^{\sigma}, \\
& \left\|\left\{\left|f_{k}\right|^{\sigma}\right\}\right\|_{\ell q / \sigma\left(L^{p / \sigma, r / \sigma}\right)}=\|f\|_{\ell^{q}\left(L^{p, r}\right)} .
\end{aligned}
$$


2.4. Real interpolation. It is well known that the Lorentz spaces occur as real interpolation spaces for the real method, namely we have $\left(L^{p_{0}}, L^{p_{1}}\right)_{\theta, r}=L^{p, r}$ if $p_{0} \neq p_{1}, 0<\theta<1$ and $(1-\theta) p_{0}^{-1}+\theta p_{1}^{-1}=p^{-1}$. This remains true for vector-valued spaces (in particular $\ell^{q}$-valued spaces), see [4, $\left.\S 5.2, \S 5.6\right]$. Finally if $\|a\|_{\ell_{s}^{q}(A)}=\left(\sum_{k=0}^{\infty} 2^{k s q}\left\|a_{k}\right\|_{A}^{q}\right)^{1 / q}$ then for vector-valued $\ell^{q}$ spaces one has $\left(\ell_{s_{0}}^{q_{0}}\left(A_{0}\right), \ell_{s_{1}}^{q_{1}}\left(A_{1}\right)\right)_{\theta, q}=\ell_{s}^{q}\left(\left(A_{0}, A_{1}\right)_{\theta, q}\right)$ for $\left(s, q^{-1}\right)=(1-\theta)\left(s_{0}, q_{0}^{-1}\right)+$ $\theta\left(s_{1}, q_{1}^{-1}\right)$, see [4, 5.6.2]. Examining e.g. arguments in [30] these observations can be used to extend some of the known characterizations of Besov and Triebel-Lizorkin spaces to their Lorentz-space based analogues.

2.5. Sums. The expression (9) is not a norm unless $1 \leq r \leq p$. It is well known that the spaces $L^{p, r}$ are normable for $p>1$ and $r \geq 1$; one replaces $f^{*}$ by the maximal function $f^{* *}$ in the definition of the Lorentz spaces to get an equivalent expression which is a norm. We write

$$
\|f\|_{p, r}=\left(\int_{0}^{\infty} t^{r / p} f^{* *}(t)^{r} \frac{d t}{t}\right)^{1 / r} .
$$

We also use $\|f\|_{L^{p, r}\left(\ell^{q}\right)},\|f\|_{\ell^{q}\left(L^{p, r}\right)}$ for the expressions corresponding to (12), but with the ${ }^{* *}$-functions. See $[12$ or 3$]$. The additivity property holds when the measure space is nonatomic, since in these cases we have a triangle inequality for

$$
f \mapsto f^{* *}(t)=\frac{1}{t} \int_{0}^{t} f^{*}(s) d s=\sup _{E: \mu(E) \leq t} \frac{1}{t} \int_{E}|f| d \mu .
$$

See [12] or [3, ch.2]. The true norms can be used to prove duality theorems; one identifies the dual of $L^{p, q}, 1<p<\infty, 1 \leq q<\infty$ with $L^{p^{\prime}, q^{\prime}}$. This also works on discrete spaces, with counting measure (see [3, ch.2.4]).

If we formulate the triangle inequality with the original quasi-norms in (9) we get for nonatomic $\mu$,

$$
\left\|\sum_{k} f_{k}\right\|_{p, r} \leq C_{p, r} \sum_{k}\left\|f_{k}\right\|_{p, r}, 1<p<\infty, r \geq 1
$$

with $C_{p, r}=C_{p}=\left(1-p^{-1}\right)^{-1}$. This is proved using the additivity of the functional in (17) in combination with Minkowski's and Hardy's inequalities ([3, p. 124]). Lorentz [16] showed that one can take $C_{p, r}=1$ for $1 \leq r \leq p$. Barza, Kolyada and Soria 2 showed for $1<p<r$ that the best constant $C_{p, r}$ in (18) is given by $(p / r)^{1 / r}\left(p^{\prime} / r^{\prime}\right)^{1 / r^{\prime}}$.

One can use (16) and (18) for the space $L^{p / u, r / u}$ to get

$$
\left\|\sum_{k} f_{k}\right\|_{p, r} \lesssim_{p, r, u}\left(\sum_{k}\left\|f_{k}\right\|_{p, r}^{u}\right)^{1 / u}, u<\min \{p, r, 1\} .
$$

However this can be improved in some cases. The analogue of (18) fails for $L^{1, r}, r>1$, (cf. [27] for a weaker substitute) but there is a different kind of 
triangle inequality for $p<1$, for the $p$ th power of $\|\cdot\|_{p, r}$, which gives

$$
\left\|\sum_{k} f_{k}\right\|_{p, r} \leq C(p, r)\left(\sum_{k}\left\|f_{k}\right\|_{p, r}^{p}\right)^{1 / p}, \quad p<1, \quad r \geq p ;
$$

here $C(p, r) \leq\left(\frac{2-p}{1-p}\right)^{1 / p}$. This was proved for $r=\infty$ by Stein, Taibleson and Weiss [25], (see also Kalton [14, and unpublished work of Pisier and Zinn mentioned in [14]). It is easy to modify the proof in 25] to cover the cases $p<r<\infty$ with the same constant. However for $r=p$ one can put of course put $C(p, p)=1$ which suggests that the behavior of $C(p, r)$ should improve for $r>p$ as $r$ decreases. We shall prove such a result in Appendix B and show that for $0<p<1, p<r<\infty$

$$
C(p, r) \leq A^{1 / p}\left(\frac{1}{1-p}\right)^{1 / p-1 / r}\left(1+\frac{p}{r} \log \frac{1}{1-p}\right)^{1 / p-1 / r}
$$

and $A$ does not depend on $p$ and $r$. The precise behavior of $C(p, r)$ is not relevant for the results in this paper, but (21) should be interesting in its own right. Note that the logarithmic term in (21) vanishes as $r \rightarrow p+$ and as $r \rightarrow \infty$.

Open problem. It would be interesting to get more precise information on $C(p, r)$, in particular one would like to know whether the logarithmic term in (21) is necessary for $p<r<\infty$.

2.5.1. Computations of some lower bounds. Suppose we are given $b>0$ and sets $A_{j}$, indexed by $j \in \mathcal{Z} \subset \mathbb{Z}$ such that

$$
\mu\left(A_{j}\right) \geq b \rho^{j}, \quad j \in \mathcal{Z}
$$

for some $\rho>1$. Assume that, for a nonnegative sequence $\beta \in \ell^{r}(\mathbb{Z})$,

$$
|f(x)| \geq \sum_{j \in \mathcal{Z}} \beta(j) \rho^{-j / p_{1}} \mathbb{A}_{j}(x) \text { a.e. }
$$

Then for $0<p<\infty, 0<r \leq \infty$,

$$
\|f\|_{p, r} \gtrsim\left(\sum_{j \in \mathcal{Z}}|\beta(j)|^{r}\right)^{1 / r}
$$

with the obvious sup-analogue for $r=\infty$. The implicit constants depend on $p, r$.

To prove the lower bound observe that the distribution function satisfies $\mu_{f}\left(\beta(j) \rho^{-\frac{j+1}{p}}\right) \geq \mu\left(A_{j}\right)>b \rho^{j-1}$ and therefore $f^{*}\left(b \rho^{j-1}\right) \geq \beta(j) \rho^{-\frac{j+1}{p}}$ by definition of the rearrangement function. This easily implies (22C), under the assumption (22b). 
2.5.2. Computations of some upper bounds. We now replace $\mathcal{Z}$ by $\mathbb{Z}$ and add the assumption that $n \mapsto \beta(n) 2^{-n / p}$ is nonincreasing. Assume that $\left\{F_{n}\right\}_{n \in \mathbb{Z}}$ is a sequence of measurable sets such that

$$
\mu\left(F_{n}\right) \leq B \rho^{n}, \quad n \in \mathbb{Z},
$$

for some $\rho>1, B>0$ and assume that,

$$
|f(x)| \leq \sum_{n \in \mathbb{Z}} \beta(n) 2^{-n / p} \mathbb{1}_{F_{n}}(x) \text { a.e. }
$$

Then for $0<p<\infty, 0<r \leq \infty$,

$$
\|f\|_{p, r} \lesssim\|\beta\|_{\ell^{r}(\mathbb{Z})} .
$$

To see this observe that

$$
\mu_{f}\left(\beta(n) 2^{-\frac{n-1}{p}}\right) \leq \mu\left(\cup_{j \leq n} F_{j}\right) \leq B \sum_{j \leq n} \rho^{j} \leq B(\rho-1)^{-1} \rho^{n+1}
$$

and therefore $f^{*}\left(\frac{B}{\rho-1} \rho^{n+1}\right) \leq \beta(n) \rho^{-\frac{n-1}{p}}$. This easily implies (23c).

\section{NECESSARY CONDITIONS}

3.1. Guide through this section. Many examples for embedding relations of spaces of Hardy-Sobolev type have been discussed in the literature (e.g [28], [21]), and most of our examples are at least related to those earlier examples.

The necessity of the condition $p_{0} \leq p_{1}$, and the necessity of the condition $r_{0} \leq r_{1}$ in the case whenever $p_{0}=p_{1}$, in all four Theorems 1.1, 1.2, 1.5, 1.6, is proved in 33.3 . The necessity of the condition $s_{0}-s_{1} \geq d / p_{0}-d / p_{1} \geq 0$ in all four theorems is proved in 33.4 .

Consider the case $s_{0}-s_{1}=d / p_{0}-d / p_{1}>0$ which is case (iii) in all four theorems. The necessity of the condition $q_{0} \leq r_{1}$ in Theorem 1.1 (iii), the necessity of the condition $r_{0} \leq q_{1}$ in Theorem 1.2 (iii), the necessity of the condition $r_{0} \leq r_{1}$ in Theorem 1.5 (iii), and the necessity of the condition $q_{0} \leq q_{1}$ in Theorem 1.6 (iii), are all proved in 3.5 .

Necessary conditions in the case $s_{0}=s_{1}$ and $p_{0}=p_{1}$. In Theorem 1.1 (iv), (v) the necessity of the condition $q_{0} \leq p_{1}$ is shown in 33.6.1, the necessity of the condition $q_{0} \leq q_{1}$ is shown in $\$ 3.6 .2$, and the necessity of the condition $q_{0} \leq r_{1}$ is shown in 93.5 . In addition, for the case $p_{1}=q_{1}<r_{0}$ in part (vi), we must have the strict inequality in $q_{0}<p_{1}$; this follows from (38) in 3.6 .3 .

In Theorem 1.2 (iv), (v) the necessity of the condition $q_{1} \geq p_{0}$ is shown in 3.6.1, the necessity of the condition $q_{0} \geq q_{1}$ is shown in 33.6.2, and the necessity of the condition $q_{1} \geq r_{0}$ is shown in 33.5 . Moreover, for the case $p_{0}=q_{0}>r_{1}$ in part (vi), we must have the strict inequality in $q_{1}>p_{0}$; this follows from (39) in 33.6 .3 . 
The necessity of the conditions $r_{0} \leq r_{1}$ in Theorems 1.5. (iv), and1.6. (iv), is shown in 3.3 (as already pointed out). The necessity of the conditions $q_{0} \leq q_{1}$ in Theorems 1.5, (iv), and 1.6, (iv), is shown in 93.6 .2 .

3.2. Preliminaries. In what follows we let $\psi_{0}$ be a $C^{\infty}$ function supported on $\{x:|x| \leq 1 / 8\}$ such that $\widehat{\psi}_{0}(\xi) \neq 0$ for $|\xi| \leq 2$ and such that

$$
\int \psi_{0}(x) d x=1, \quad \int \psi_{0}(x) x_{1}^{\nu_{1}} \ldots x_{d}^{\nu_{d}} d x=0,
$$

$$
\text { for multiindices } \nu \text { with } \nu_{i} \geq 0,0<\sum_{i=1}^{d} \nu_{i} \leq M \text {. }
$$

Here we assume that $M$ is large, specifically given $p, q, s$, the condition

$$
M>|s|+100 d \max \{1,1 / p, 1 / q\}
$$

will certainly be sufficient for our purposes. Let

$$
\begin{gathered}
\psi_{k}=2^{k d} \psi_{0}\left(2^{k} \cdot\right)-2^{(k-1) d} \psi_{0}\left(2^{k-1} \cdot\right), \quad k \geq 1, \\
\mathcal{L}_{k} f=\psi_{k} * f
\end{gathered}
$$

We can arrange $\psi_{0}$ so that $\psi_{1}$ satisfies

$$
\psi_{1} * \psi_{1}(x) \geq c \text { for } x \in I=(-\varepsilon, \varepsilon)^{d}
$$

for some fixed $\varepsilon>0$. We have (using Littlewood-Paley decompositions generated by dilates of compactly supported functions, aka local means in [30])

$$
\begin{aligned}
\|f\|_{B_{q}^{s}\left[L^{p, r}\right]} & \approx\left(\sum_{k=0}^{\infty} 2^{k s q}\left\|\mathcal{L}_{k} f\right\|_{p, r}^{q}\right)^{1 / q}, \\
\|f\|_{F_{q}^{s}\left[L^{p, r}\right]} & \approx\left\|\left(\sum_{k=0}^{\infty} 2^{k s q}\left|\mathcal{L}_{k} f\right|^{q}\right)^{1 / q}\right\|_{p, r} .
\end{aligned}
$$

These equivalences follow by modifying the corresponding arguments for the case $p=r(c f$. [30]) and combining them with real interpolation arguments, cf. 2.4 , We omit the details.

We will repeatedly need the following straightforward lemma.

Lemma 3.1. Let $W$ be a finite collection of points in $\mathbb{R}^{d}$, with mutual distance at least $2^{-n}$. Define $h(x)=\sum_{w \in W} \psi\left(2^{n}(x-w)\right)$. Then

$$
\left\|\mathcal{L}_{j} h\right\|_{\infty} \lesssim \begin{cases}2^{(n-j)(M+1)} & \text { if } j \geq n \\ 2^{(j-n)(M+1-d)} & \text { if } n \geq j\end{cases}
$$

Moreover,

$$
\left\|\mathcal{L}_{j} h\right\|_{p, r} \lesssim 2^{(n-j) M}\left(2^{-n d} \# W\right)^{1 / p}, \quad \text { if } j \geq n .
$$

and for $j=n$ we have the equivalence

$$
\left\|\mathcal{L}_{n} h\right\|_{p, r} \approx\left(2^{-n d} \# W\right)^{1 / p} .
$$


For $j \leq n$ let $W(j)$ be any maximal $2^{-j}$-separated subset of $W$. Then

$$
\left\|\mathcal{L}_{j} h\right\|_{p, r} \lesssim 2^{(j-n)(M-d)}\left(2^{-j d} \# W(j)\right)^{1 / p}, \quad \text { if } j \leq n .
$$

Proof. Note that the upper bounds need to be proved only for $p=r$ as they follow then for all $r$ by real interpolation. Let $h_{w}=\psi\left(2^{n}(\cdot-w)\right)$. The derivation is straightforward; we use the moment condition on the convolution kernel $\psi_{j}$ for the operator $\mathcal{L}_{j}$ to bound $\left|\mathcal{L}_{j} h_{w}(x)\right| \lesssim 2^{(n-j)(M+1)}$ for $j>n$. The corresponding $L^{p}$ bound follows as the supports of $\mathcal{L}_{j} h_{w}$ are essentially disjoint. When $j \leq n$ we use the moment condition on $h_{w}$ to bound $\left|\mathcal{L}_{j} h_{w}(x)\right| \lesssim 2^{(j-n)(M+1)}$. The bound for $\mathcal{L}_{j} h$ follows since $\mathcal{L}_{j} h_{w}(x)$ is nonzero for at most $O\left(2^{(n-j) d}\right.$ ) terms (and one gets improvements for sparse $W)$. The corresponding $L^{p}$ bound is then an immediate consequence.

In order to obtain the lower bound for $\left\|\mathcal{L}_{n} h\right\|_{p, r}$ we use the assumption (26) to see that $\left|\mathcal{L}_{n} h\right| \geq c$ on a set of measure $2^{-n d} \# W$.

In what follows we shall denote by $B(x, \rho)$ the ball of radius $\rho$ centered at $x$.

3.3. Necessity of $p_{0} \leq p_{1}$, and of $r_{0} \leq r_{1}$ in the case $p_{0}=p_{1}$. Let $\psi_{1}$ be as in $\$ 3.2, e_{1}=(1,0, \ldots, 0\}$, and let

$$
f(x)=\sum_{n=1}^{\infty} a_{n} \psi_{1}\left(x-n e_{1}\right) .
$$

It is easy to see by (28) and Minkowski's inequality that for any $M$

$$
\left|\mathcal{L}_{k} f(x)\right| \lesssim 2^{-k M} \sum_{n=1}^{\infty}\left|a_{n}\right| \mathbb{1}_{B\left(n e_{1}, 1\right)}(x) .
$$

This implies $\left\|\mathcal{L}_{k} f\right\|_{p, r} \lesssim 2^{-k M}\|a\|_{\ell^{p}}$. We also have

$$
\left|\mathcal{L}_{1} f(x)\right|=\left|\sum_{n=1}^{\infty} a_{n} \psi_{1} * \psi_{1}\left(x-n e_{1}\right)\right| \geq \sum_{n=1}^{\infty}\left|a_{n}\right| \mathbb{1}_{B\left(n e_{1}, \varepsilon\right)}(x)
$$

which implies the lower bound $\left\|\mathcal{L}_{1} f\right\|_{p, r} \gtrsim\|a\|_{\ell p, r}$. It follows that $p_{0} \leq p_{1}$ in all cases.

The same calculation proves the necessary condition $r_{0} \leq r_{1}$ in the case $p_{0}=p_{1}$.

3.4. Necessity of $s_{0}-s_{1} \geq d\left(1 / p_{0}-1 / p_{1}\right)$. Let $\chi \in C_{c}^{\infty}\left(\mathbb{R}^{d}\right)$ be supported in the ball of radius $10^{-2}$ centered at 1 . Let $\beta_{k}$ be as in the definition of $\Lambda_{k}$ in the introduction, so that for $k \geq 1$ we have $\beta_{k}(\xi)=0$ when $2^{-k}|\xi| \notin\left[\frac{3}{4}, \frac{7}{4}\right]$ and $\beta_{k}(\xi)=1$ when $2^{-k}|\xi| \in\left[\frac{7}{8}, \frac{3}{2}\right]$. Let $\omega_{k}=2^{k d} \mathcal{F}^{-1}[\chi]\left(2^{k} x\right)$ and notice that $\Lambda_{k} \omega_{k}=\omega_{k}$ and $\Lambda_{j} \omega_{k}=0$ when $j \neq k$. We have by scaling $\left\|\Lambda_{k} \omega_{k}\right\|_{p, r}=$ $2^{k(d-d / p)}\left\|\mathcal{F}^{-1}[\chi]\right\|_{p, r}$ and thus any of the embeddings in the four theorems in the introduction requires $2^{k\left(s_{1}-d / p_{1}\right)} \lesssim 2^{k\left(s_{0}-d / p_{0}\right)}$ for $k \geq 0$. Hence $s_{0}-s_{1} \geq d\left(1 / p_{0}-1 / p_{1}\right)$. 
3.5. Necessary conditions for the case $s_{0}-s_{1}=d\left(1 / p_{0}-1 / p_{1}\right) \geq 0$. Let $R \gg 8$ be large and let $\left\{n_{l}\right\}_{l=1}^{\infty}$ be an increasing sequence of integers which is sufficiently separated, i.e. such that $n_{l} \gg l \geq R, n_{l+1}-n_{l} \geq R$. Let $\mathfrak{N}:=$ $\left\{n_{l}: l \in \mathbb{N}\right\}$. Let $\left\{a_{l}\right\}_{l=1}^{\infty}$ be a decreasing sequence for which $l \mapsto 2^{n_{l} d / p}\left|a_{l}\right|$ is increasing. Define $\Psi_{n}(x):=\psi_{1}\left(2^{n}\left(x-2^{-n} e_{1}\right)\right)$, with $\psi_{1}$ as in 83.2 and

$$
h_{\gamma}(x)=\sum_{l=1}^{\infty} a_{l} 2^{n_{l} \gamma} \Psi_{n_{l}}(x) .
$$

Lemma 3.2. Let $s \in \mathbb{R}$. If the separation constant $R$ in the definition of $\mathfrak{N}$ is sufficiently large then

$$
\begin{aligned}
\left\|h_{-s+d / p}\right\|_{B_{q}^{s}\left[L^{\left.p, r_{0}\right]}\right.} & \approx\|a\|_{\ell q}, \\
\left\|h_{-s+d / p}\right\|_{F_{\rho}^{s}\left[L^{p, r}\right]} & \approx\|a\|_{\ell^{r} .}
\end{aligned}
$$

As an immediate consequence we get

Corollary 3.3. Suppose $s_{0}-s_{1}=d / p_{0}-d / p_{1} \geq 0$. Then we have the following implications

$$
\begin{aligned}
& B_{q_{0}}^{s_{0}}\left[L^{p_{0}, r_{0}}\right] \hookrightarrow B_{q_{1}}^{s_{1}}\left[L^{p_{1}, r_{1}}\right] \Longrightarrow q_{0} \leq q_{1}, \\
& F_{q_{0}}^{s_{0}}\left[L^{p_{0}, r_{0}}\right] \hookrightarrow F_{q_{1}}^{s_{1}}\left[L^{p_{1}, r_{1}}\right] \Longrightarrow r_{0} \leq r_{1}, \\
& B_{q_{0}}^{s_{0}}\left[L^{p_{0}, r_{0}}\right] \hookrightarrow F_{q_{1}}^{s_{1}}\left[L^{p_{1}, r_{1}}\right] \Longrightarrow q_{0} \leq r_{1}, \\
& F_{q_{0}}^{s_{0}}\left[L^{p_{0}, r_{0}}\right] \hookrightarrow B_{q_{1}}^{s_{1}}\left[L^{p_{1}, r_{1}}\right] \Longrightarrow r_{0} \leq q_{1} .
\end{aligned}
$$

Proof of Lemma 3.2, Let $u<\min \left\{p, q, r_{0}\right\}$. By (19) we have

$$
\left\|h_{\gamma}\right\|_{B_{q}^{s}\left[L^{p, r_{0}}\right]} \lesssim\left(\sum_{j=0}^{\infty} 2^{j s q}\left(\sum_{l=1}^{\infty}\left\|a_{l} 2^{n_{l} \gamma} \mathcal{L}_{j} \Psi_{n_{l}}\right\|_{p, r_{0}}^{u}\right)^{q / u}\right)^{1 / q} .
$$

We use Lemma 3.1 for a singleton $W$ to estimate for $\gamma=-s+d / p$, the right hand side in the last display by a constant times

$$
\begin{aligned}
& \left(\sum_{j=0}^{\infty} 2^{j s q}\left(\sum_{l=1}^{\infty}\left|a_{l}\right|^{u} 2^{n_{l}(\gamma-d / p) u} 2^{-\left|j-n_{l}\right|(M-2 d) u}\right)^{q / u}\right)^{1 / q} \\
& \lesssim\left(\sum_{j=0}^{\infty}\left(\sum_{l=1}^{\infty}\left|a_{l}\right|^{u} 2^{-\left|j-n_{l}\right|(M-2 d-|s|) u}\right)^{q / u}\right)^{1 / q} \\
& \lesssim\left(\sum_{j=0}^{\infty} \sum_{l=1}^{\infty}\left|a_{l}\right|^{q} 2^{-\left|j-n_{l}\right| q}\right)^{1 / q} \lesssim\left(\sum_{l=1}^{\infty}\left|a_{l}\right|^{q}\right)^{1 / q} ;
\end{aligned}
$$

in this calculation we have used $M-2 d-|s|>1$. We have proved the upper bound in (31).

For the lower bound we estimate

$$
\left\|h_{-s+d / p}\right\|_{B_{q}^{s}\left[L^{p, r_{0}}\right]} \gtrsim\left(\sum_{k=1}^{\infty} 2^{n_{k} s q}\left\|\mathcal{L}_{n_{k}} h_{-s+d / p}\right\|_{p, r_{0}}^{q}\right)^{1 / q} \geq c I-C I I
$$


where

$$
\begin{aligned}
I & =\left(\sum_{k=1}^{\infty}\left|a_{k}\right|{ }^{q} 2^{n_{k} d / p}\left\|\mathcal{L}_{n_{k}} \Psi_{n_{k}}\right\|_{p, r_{0}}^{q}\right)^{1 / q}, \\
I I & =\left(\sum_{k=1}^{\infty} 2^{n_{k} s q}\left\|\sum_{\substack{l \geq 1: \\
l \neq k}} a_{l} 2^{-n_{l} s} 2^{n_{l} d / p} \mathcal{L}_{n_{k}} \Psi_{n_{l}}\right\|_{p, r_{0}}^{q}\right)^{1 / q} .
\end{aligned}
$$

We have (using (26) ) $2^{n_{k} d / p}\left\|\mathcal{L}_{n_{k}} \Psi_{n_{k}}\right\|_{p, r_{0}} \geq c>0$ uniformly in $k$ and therefore $I \gtrsim\|a\|_{\ell q}$. The above computation for the upper bound also gives $I I \lesssim 2^{-R}\|a\|_{\ell^{q}}$ and the lower bound in (31) follows if $R$ is chosen sufficiently large.

We now turn to the proof of (32). For the upper bound we may assume without loss of generality that $\rho<\min \{1, r, p\}$. Then by Lemma 3.1

$$
\left(\sum_{j=0}^{\infty}\left|2^{j s} \sum_{l=1}^{\infty} a_{l} 2^{n_{l}\left(\frac{d}{p}-s\right)} \mathcal{L}_{j} \Psi_{n_{l}}(x)\right|^{\rho}\right)^{1 / \rho} \leq \mathcal{E}_{1}(x)+\mathcal{E}_{2}(x)
$$

where

$$
\begin{aligned}
& \mathcal{E}_{1}(x)=\left(\sum_{j=0}^{\infty} 2^{j s \rho} \sum_{\substack{l \in \mathbb{N} \\
n_{l} \leq j}}\left|a_{l}\right|^{\rho} 2^{n_{l}\left(\frac{d}{p}-s\right) \rho} 2^{-\left(j-n_{l}\right)(M+1) \rho} \mathbb{1}_{B\left(0,2^{1-n_{l}}\right)}(x)\right)^{1 / \rho} \\
& \mathcal{E}_{2}(x)=\left(\sum_{j=0}^{\infty} 2^{j s \rho} \sum_{\substack{l \in \mathbb{N} \\
n_{l}>j}}\left|a_{l}\right|^{\rho} 2^{n_{l}\left(\frac{d}{p}-s\right) \rho} 2^{-\left(n_{l}-j\right)(M+1-d) \rho} \mathbb{1}_{B\left(0,2^{1-j}\right)}(x)\right)^{1 / \rho} .
\end{aligned}
$$

Interchanging the $n_{l}, j$ summations and summing a geometric series (where $M+1>|s|)$ yields

$$
\mathcal{E}_{1}(x) \leq\left(\sum_{l=1}^{\infty}\left|a_{l}\right|^{\rho} 2^{n_{l} \frac{d}{p} \rho_{1}} \mathbb{1}_{B\left(0,2^{\left.1-n_{l}\right)}\right.}(x)\right)^{1 / p}
$$

and, with the parameter $m=n_{l}-j$,

$$
\mathcal{E}_{2}(x) \leq\left(\sum_{m=0}^{\infty} 2^{-m\left(M+1-d+s-\frac{d}{p}\right) \rho} \mathcal{E}_{2, m}(x)^{\rho}\right)^{1 / \rho}
$$

where

$$
\mathcal{E}_{2, m}(x)=\left(\sum_{\substack{l \in \mathbb{N}: \\ n l \geq m}}\left|a_{l}\right|^{\rho} 2^{\left(n_{l}-m\right) \frac{d}{p} \rho_{1}} \mathbb{1}_{B\left(0,2^{1-n_{l}+m}\right)}(x)\right)^{1 / \rho} .
$$

We use (15), and 92.5 .2 with the parameter $\rho=2^{-d}$ and with exponents $(p / \rho, r / \rho)$ in place of $(p, r)$, to get

$$
\left\|\mathcal{E}_{1}\right\|_{p, r}=\left\|\mathcal{E}_{1}^{\rho}\right\|_{p / \rho, r / \rho}^{1 / \rho} \lesssim\|a\|_{\ell^{r}} .
$$

Similarly $\left\|\mathcal{E}_{2, m}\right\|_{p, r} \lesssim\|a\|_{\ell^{r}}$ uniformly in $m$, and then from (34) we also get $\left\|\mathcal{E}_{2}\right\|_{p, r} \lesssim\|a\|_{\ell^{r}}$. 
For the lower bound

$$
\begin{aligned}
& \left(\sum_{j=0}^{\infty}\left|2^{j s} \sum_{l=1}^{\infty} a_{l} 2^{n_{l}\left(\frac{d}{p}-s\right)} \mathcal{L}_{j} \Psi_{n_{l}}(x)\right|^{\rho}\right)^{1 / \rho} \\
& \geq\left(\sum_{k=1}^{\infty}\left|2^{n_{k} s} \sum_{l=1}^{\infty} a_{l} 2^{n_{l}\left(\frac{d}{p}-s\right)} \mathcal{L}_{n_{k}} \Psi_{n_{l}}(x)\right|^{\rho}\right)^{1 / \rho} \geq \mathcal{E}_{3}(x)-\mathcal{E}_{4}(x)
\end{aligned}
$$

where

$$
\begin{aligned}
& \mathcal{E}_{3}(x)=\left(\sum_{k=1}^{\infty}\left|a_{k} 2^{n_{k} \frac{d}{p}} \mathcal{L}_{n_{k}} \Psi_{n_{k}}(x)\right|^{\rho}\right)^{1 / \rho} \\
& \mathcal{E}_{4}(x)=\left(\sum_{k=1}^{\infty}\left|2^{n_{k} s} \sum_{\substack{l \in \mathbb{N}: \\
l \neq k}} a_{l} 2^{n_{l}\left(\frac{d}{p}-s\right)} \mathcal{L}_{n_{k}} \Psi_{n_{l}}(x)\right|^{\rho}\right)^{1 / \rho} .
\end{aligned}
$$

Note that $\mathcal{L}_{n_{k}} \Psi_{n_{k}}(x)=\psi_{1} * \psi_{1}\left(2^{n_{k}} x-e_{1}\right)$ and thus $\left|\mathcal{L}_{n_{k}} \Psi_{n_{k}}(x)\right| \geq c>0$ on $B\left(2^{-n_{k}} e_{1}, 2^{-n_{k}} \varepsilon\right)$. Hence

$$
\mathcal{E}_{3}(x) \geq c\left(\sum_{k=1}^{\infty}\left|a_{k} 2^{n_{k} \frac{d}{p}} \mathbb{1}_{B\left(2^{\left.-n_{k} e_{1}, 2^{-n} n_{k \varepsilon}\right)}\right.}(x)\right|^{\rho}\right)^{1 / \rho},
$$

which by 2.5.1 implies $\left\|\mathcal{E}_{3}\right\|_{p, r} \gtrsim\|a\|_{\ell^{r}}$. Analyzing the proof of the upper bound and taking into account the $R$-separation of the numbers $n_{l}$ yields $\left\|\mathcal{E}_{4}\right\|_{p, r} \lesssim 2^{-R}\|a\|_{\ell^{r}}$ and for large $R$ we get the lower bound in (32).

3.6. The case $s_{0}=s_{1}=s, p_{0}=p_{1}=p$.

3.6.1. Necessary conditions on $\left(p, q_{0}\right)$ and $\left(p, q_{1}\right)$ in Theorems 1.1. 1.2. Let $R \gg 8$ be large and $\mathfrak{N}=\left\{n_{l}: l \in \mathbb{N}\right\}$ be as in $\oint 3.5$, i.e. $l \mapsto n_{l}$ is increasing and $R$-separated.

Lemma 3.4. Let $a=\{a\}_{l=1}^{\infty}$ be a sequence such that $l \mapsto\left|a_{l}\right|$ is nonincreasing. Let, for $\nu \in \mathbb{Z}^{d}, g_{l, \nu}(x)=\psi\left(2^{n_{l}}\left(x-\left(l e_{1}+2^{-n_{l}} \nu\right)\right)\right)$ and let

$$
g_{l}(x)=\sum_{\substack{2^{3} \leq \nu_{i} \leq 2^{n_{l}-3} \\ i=1, \ldots, d}} g_{l, \nu}(x) .
$$

Define $f(x)=\sum_{l=1}^{\infty} 2^{-s n_{l}} a_{l} g_{l}(x)$. Then

$$
\begin{aligned}
\|f\|_{B_{q}^{s}\left[L^{p, r}\right]} & \approx\left(\sum_{l=1}^{\infty}\left|a_{l}\right|^{q}\right)^{1 / q}, \\
\|f\|_{F_{q}^{s}\left[L^{p, r}\right]} & \approx\left(\sum_{l=1}^{\infty} l^{\frac{r}{p}-1}\left|a_{l}\right|^{r}\right)^{1 / r},
\end{aligned}
$$

provided that the separation constant $R$ is large enough. 
Note that the expression of the right hand side of (36) is equivalent to the $\ell^{p, r}$ norm of $a$ (if $a$ is nonincreasing).

The lemma implies the following corollary relevant for Theorems 1.1 and 1.2 .

\section{Corollary 3.5.}

$$
\begin{aligned}
& B_{q_{0}}^{s}\left[L^{p, r_{0}}\right] \hookrightarrow F_{q_{1}}^{s}\left[L^{p, r_{1}}\right] \Longrightarrow q_{0} \leq p . \\
& F_{q_{0}}^{s}\left[L^{p, r_{0}}\right] \hookrightarrow B_{q_{1}}^{s}\left[L^{p, r_{1}}\right] \Longrightarrow p \leq q_{1} .
\end{aligned}
$$

Proof of Lemma 3.4. By Lemma 3.1

$$
\begin{aligned}
\left\|\mathcal{L}_{j} g_{l}\right\|_{p, r} & \lesssim 2^{-\left|n_{l}-j\right|(M-d)}, \\
\left\|\mathcal{L}_{n_{l}} g_{l}\right\|_{p, r} & \approx 1 .
\end{aligned}
$$

We first establish the upper bound in (35) and let $u<\min \{p, q, r\}$. Estimate using (19), (37a) and then Hölder's inequality

$$
\begin{aligned}
\|f\|_{B_{q}^{s}\left[L^{p, r}\right]} & \lesssim\left(\sum_{j=0}^{\infty} 2^{j s q}\left(\sum_{l=1}^{\infty}\left|a_{l}\right|^{u} 2^{-s n_{l} u}\left\|\mathcal{L}_{j} g_{l}\right\|_{p, r}^{u}\right)^{q / u}\right)^{1 / q} \\
& \lesssim\left(\sum_{j=0}^{\infty}\left(\sum_{l=1}^{\infty}\left|a_{l}\right|^{u} 2^{-(M-d-|s|)\left|j-n_{l}\right| u}\right)^{q / u}\right)^{1 / q} \\
& \lesssim\left(\sum_{j=0}^{\infty} \sum_{l=1}^{\infty}\left|a_{l}\right|^{q} 2^{-\left|j-n_{l}\right| q}\right)^{1 / q} \lesssim\left(\sum_{l=1}^{\infty}\left|a_{l}\right|^{q}\right)^{1 / q}
\end{aligned}
$$

where we used $M-d-|s|>1$.

For the lower bound in (35) we have

$$
\begin{aligned}
\|f\|_{B_{q}^{s}\left[L^{p, r}\right]} & \gtrsim\left(\sum_{k=1}^{\infty} 2^{n_{k} s q}\left\|a_{k} 2^{-s n_{k}} \mathcal{L}_{n_{k}} g_{n_{k}}+\sum_{\substack{l \in \mathbb{N}: \\
l \neq k}} a_{l} 2^{-s n_{l}} \mathcal{L}_{n_{k}} g_{n_{l}}\right\|_{p, r}^{q}\right)^{1 / q} \\
& \geq c_{I} I-C_{I I} I I
\end{aligned}
$$

where

$$
\begin{gathered}
I=\left(\sum_{k=1}^{\infty}\left|a_{k}\right|^{q}\left\|\mathcal{L}_{n_{k}} g_{n_{k}}\right\|_{p, r}^{q}\right)^{1 / q}, \\
I I \leq\left(\sum_{k=1}^{\infty} 2^{n_{k} s q}\left\|\sum_{\substack{l \in \mathbb{N}: \\
l \neq k}} a_{l} 2^{-s n_{l}} \mathcal{L}_{n_{k}} g_{n_{l}}\right\|_{p, r}^{q}\right)^{1 / q} .
\end{gathered}
$$

By (37b) we get $I \gtrsim\|a\|_{\ell^{q}}$. Using the argument for the upper bound given above and taking account (37a) with $j=n_{k} \neq n_{l}$ yields $I I \lesssim 2^{-R}\|a\|_{\ell q}$. Thus if $R$ is large enough we obtain the lower bound in (35). 
We now prove the upper bound in (36). By the $L^{\infty}$ bounds in Lemma 3.1 we have

$$
\left(\sum_{j=0}^{\infty} 2^{-j s q}\left|\mathcal{L}_{j} f(x)\right|^{q}\right)^{1 / q} \leq C G(x)
$$

where $G(x)=\sum_{l=1}^{\infty}\left|a_{l}\right| \mathbb{1}_{Q_{l}}(x)$, with $Q_{l}=l+[-1 / 4,1 / 4]^{d}$. The rearrangement of $G$ satisfies

$$
G^{*}(t) \leq \sum_{l=1}^{\infty}\left|a_{l}\right| \mathbb{1}_{\left((l-1) 2^{-d}, l 2^{-d}\right]}(t)
$$

and we obtain

$$
\|f\|_{F_{q}^{s}\left[L^{p, r}\right]} \lesssim\left(\frac{r}{p} \int_{0}^{\infty}\left[t^{1 / p} G^{*}(t)\right]^{r} \frac{d t}{t}\right)^{1 / r} \lesssim\left(\sum_{l=1}^{\infty} l^{\frac{r}{p}-1}\left|a_{l}\right|^{r}\right)^{1 / r} .
$$

For the lower bound we estimate

$$
\|f\|_{F_{q}^{s}\left[L^{(p, r)}\right]} \geq\left\|\left(\sum_{l} 2^{n_{l} s q}\left|\mathcal{L}_{n_{l}} f\right|^{q}\right)^{1 / q}\right\|_{L^{p, r}} \geq c I^{\prime}-C I I^{\prime}
$$

where

$$
\begin{aligned}
I^{\prime} & =\left\|\left(\sum_{l=1}^{N}\left|a_{l} \mathcal{L}_{n_{l}} g_{n_{l}}\right|^{q}\right)^{1 / q}\right\|_{p, r}, \\
I I^{\prime} & =\left\|\left(\sum_{l=1}^{N} 2^{n_{l} s q}\left|\sum_{\substack{k \geq 1: \\
k \neq l}} a_{k} 2^{-s n_{k}} \mathcal{L}_{n_{l}} g_{n_{k}}\right|^{q}\right)^{1 / q}\right\|_{p, r} .
\end{aligned}
$$

Let $\varepsilon$ be as in (26) and $J_{l, \nu, \varepsilon}=\left\{x:\left|x-l e_{1}-\nu 2^{-n_{l}}\right| \leq 2^{-n_{l}} \varepsilon\right\}$. Let $J_{l, \varepsilon}$ be the union of the $J_{l, \nu, \varepsilon}$ over all $\nu \in \mathbb{Z}^{d}$ with $8 \leq \nu_{i} \leq 2^{n_{l}-3}$ for $i=1, \ldots, d$. Notice that $J_{l, \varepsilon}$ is contained in a cube of sidelength $1 / 2$ centered at $l e_{1}$. By the condition (26) ) we have $\left|\mathcal{L}_{n_{l}} g_{n_{l}, \nu}(x)\right| \geq c$ for $x \in J_{l, \nu, \varepsilon}$. We have

$$
\left(\sum_{l=1}^{N}\left|a_{l} \mathcal{L}_{n_{l}} g_{n_{l}}(x)\right|^{q}\right)^{1 / q} \geq G_{\text {low }}(x)
$$

where

$$
G_{\text {low }}(x)=\sum_{l=1}^{N}\left|a_{l}\right| \sum_{\nu=2^{3}}^{2^{n_{l}-3}} \mathbb{1}_{J_{l, \nu, \varepsilon}}(x) .
$$

Note that the measure of $J_{l, \varepsilon}$ is at least $c_{0} \varepsilon^{d}$ for some fixed positive $c_{0}$. Hence

and thus

$$
G_{\text {low }}^{*}(t) \geq \sum_{l=1}^{N}\left|a_{l}\right| \mathbb{1}_{\left(c_{0} \varepsilon^{d}(l-1), c_{0} \varepsilon^{d} l\right]}(t)
$$

$$
I^{\prime} \geq\left\|G_{\mathrm{low}}\right\|_{L^{p, r}} \geq c^{\prime}\left(\sum_{l=1}^{N} l^{\frac{r}{p}-1}\left|a_{l}\right|^{r}\right)^{1 / r} .
$$


For $I I^{\prime}$ we get a better upper bound. By the argument for the upper bound above we obtain due to separateness condition of the $n_{l}$

$$
I I^{\prime} \leq C 2^{-R}\left(\sum_{l=1}^{N} l^{\frac{r}{p}-1}\left|a_{l}\right|^{r}\right)^{1 / r} .
$$

Thus if $R$ in our definition of the $n_{l}$ is chosen large enough we get the lower bound

$$
\|f\|_{F_{q}^{s}\left[L^{p, r}\right]} \geq c^{\prime \prime}\left(\sum_{l=1}^{N} l^{\frac{r}{p}-1}\left|a_{l}\right|^{r}\right)^{1 / r}
$$

provided that the right hand side is finite.

3.6.2. Conditions on $q_{0}, q_{1}$ when $p_{0}=p_{1}, s_{0}=s_{1}$. Let $\chi \in C_{c}^{\infty}\left(\mathbb{R}^{d}\right)$ be supported in $\left\{\xi:|\xi| \leq 2^{-4}\right\}$ such that $\chi(0)=1$, and $\widehat{\chi}(0)=1$. Let, given $N \in \mathbb{N}, f(x)=\sum_{l=N+1}^{2 N} 2^{-l s} a_{l} \eta_{l}(x)$ with $\eta_{l}=\mathcal{F}^{-1}\left[\chi\left(\cdot-2^{l} e_{1}\right)\right]$. Using the support properties of $\beta_{k}$ (cf. \$3.4) we have, for $l>1, \Lambda_{l} \eta_{l}=\eta_{l}$ and $\Lambda_{k} \eta_{l}=0$ for $k \neq l$. Hence for large $N$

$$
\begin{aligned}
\|f\|_{B_{q_{0}}^{s}\left[L^{p, r}\right]} & \approx\left(\sum_{l=N+1}^{2 N}\left|a_{l}\right|^{q_{0}}\right)^{1 / q_{0}}, \\
\|f\|_{F_{q_{1}}^{s}\left[L^{p, r}\right]} & \approx\left(\sum_{l=N+1}^{2 N}\left|a_{l}\right|^{q_{1}}\right)^{1 / q_{1}} .
\end{aligned}
$$

This immediately yields that every of the embeddings $B_{q_{0}}^{s}\left[L^{p, r_{0}}\right] \hookrightarrow F_{q_{1}}^{s}\left[L^{p_{1}, r_{1}}\right]$, $F_{q_{0}}^{s}\left[L^{p, r_{0}}\right] \hookrightarrow B_{q_{1}}^{s}\left[L^{p_{1}, r_{1}}\right], B_{q_{0}}^{s}\left[L^{p, r_{0}}\right] \hookrightarrow B_{q_{1}}^{s}\left[L^{p_{1}, r_{1}}\right], B_{q_{0}}^{s}\left[L^{p, r_{0}}\right] \hookrightarrow B_{q_{1}}^{s}\left[L^{p_{1}, r_{1}}\right]$ implies that $q_{0} \leq q_{1}$.

3.6.3. Necessary conditions on $\left(p, r_{0}\right)$ and $\left(p, r_{1}\right)$ in Theorems 1.1, 1.2, (vi). We now show

$$
\begin{aligned}
& B_{p}^{s}\left[L^{p, r_{0}}\right] \hookrightarrow F_{p}^{s}\left[L^{p, r_{1}}\right] \Longrightarrow r_{0} \leq p . \\
& F_{p}^{s}\left[L^{p, r_{0}}\right] \hookrightarrow B_{p}^{s}\left[L^{p, r_{1}}\right] \Longrightarrow p \leq r_{1} .
\end{aligned}
$$

For both implications we choose large parameters $R, N \in \mathbb{N}$ and use as a test function

$$
f(x)=\sum_{k=N+1}^{2 N} 2^{-s R k} \sum_{l=0}^{4 N}(1+|R k-R l|)^{-\frac{1}{p}}(\log (2+|R k-R l|))^{-\delta} f_{k, l}(x),
$$

where

$$
f_{k, l}(x)=\mathcal{F}^{-1}\left[\chi\left(\cdot-2^{R k} e_{1}\right)\right]\left(x-R l e_{1}\right),
$$

with $\chi$ as in 3.6 .2 .

The parameter $\delta$ will be suitably chosen depending on the parameters $r_{0}, p$ or $r_{1}, p$ in (38), (39), respectively. Let $\varepsilon>0$ such that

$$
\left|\mathcal{F}^{-1}[\chi](x)\right| \geq c>0 \text { for } x \in[-\varepsilon, \varepsilon]^{d} .
$$


We have $\Lambda_{R k} f_{k, l}=f_{k, l}$, and $\Lambda_{j} f_{k, l}=0$ when $j \neq R k$. Let $\eta=\mathcal{F}^{-1}[\chi]$ then $f_{k, l}(x)=\eta\left(x-R l e_{1}\right) e^{2 \pi i 2^{R k} x_{1}}$. Hence

$$
\begin{array}{r}
2^{s R k}\left|\Lambda_{R k} f(x)\right|=\left|\sum_{l \in \mathbb{Z}}(1+|R k-R l|)^{-\frac{1}{p}}(\log (2+R|k-l|))^{-\delta} \eta\left(x-R l e_{1}\right)\right| \\
\text { if } N+1 \leq k \leq 2 N
\end{array}
$$

and $2^{s j}\left|\Lambda_{j} f(x)\right|=0$ if $j \notin R \mathbb{N}$ or if $j \notin[R(N+1), 2 R N]$.

Proof of (38). We argue by contradiction and assume $r_{0}>p$. Choose $\delta$ so that $1 / r_{0}<\delta<1 / p$ and let $f$ be as in (40). We then have for fixed $k$ that

$$
\left\|\sum_{l \in \mathbb{Z}}(1+|R k-R l|)^{-\frac{1}{p}}(\log (2+R|k-l|))^{-\delta} \eta\left(\cdot-R l e_{1}\right)\right\|_{p, r_{0}} \lesssim 1
$$

and hence

$$
\|f\|_{B_{p}^{s}\left(L^{p, r_{0}}\right)} \lesssim N^{1 / p}
$$

To derive a lower bound for $\|f\|_{F_{p}^{s}\left[L^{p, \infty}\right]}$ we let

$$
\mathcal{U}_{\varepsilon}=\left\{x:\left|x-k_{0} R e_{1}\right| \leq \varepsilon \text { for some } k_{0} \in[5 N / 4,7 N / 4]\right\} .
$$

Fix $x \in \mathcal{U}_{\varepsilon}$. Then $\left(\sum_{j}\left|2^{j s} \Lambda_{j} f(x)\right|^{p}\right)^{1 / p} \geq c_{1} I(x)-c_{2} I I(x)$ where

$$
I(x)=c\left(\sum_{k=N+1}^{2 N}\left(1+\left|R k e_{1}-x\right|\right)^{-1}\left(\log \left(2+\left|R k e_{1}-x\right|\right)\right)^{-\delta p}\right)^{1 / p}
$$

and $I I(x)=$

$$
C\left(\sum_{k=N+1}^{2 N}\left|\sum_{l \neq k}(1+R|k-l|)^{-1 / p}(\log (2+R|k-l|))^{-\delta} \eta\left(x-R l e_{1}\right)\right|^{p}\right)^{1 / p} .
$$

Now $I(x) \gtrsim\left(R^{-1} \sum_{2 \leq l \leq N / 4} l^{-1}(\log l)^{-\delta p}\right)^{1 / p} \gtrsim R^{-1 / p}(\log N)^{\frac{1-\delta p}{p}}$, as $\delta p<1$.

Using the decay of $\eta$ we also get $I I(x) \lesssim C_{N_{1}} R^{-N_{1}}(\log N)^{\frac{1-\delta p}{p}}$ for any $N_{1}>0$. Hence for $R$ large we see that the measure of the subset of $\mathcal{U}_{\varepsilon}$ where $\left(\sum_{j}\left|2^{j s} \Lambda_{j} f(x)\right|^{p}\right)^{1 / p} \geq c(\log N)^{\frac{1-\delta p}{p}}$ is bounded below by times $c \varepsilon^{d} N$. Hence

$$
\|f\|_{F_{p}^{s}\left(L^{\left.p, r_{1}\right)}\right.} \gtrsim\|f\|_{F_{p}^{s}\left(L^{p, \infty}\right)} \gtrsim \varepsilon N^{1 / p}(\log N)^{\frac{1-\delta p}{p}} .
$$

Comparing (41) and (42), and choosing $N$ large, we get a contradiction. Hence $r_{0} \leq p$.

Proof of (39). Again we argue by contradiction and assume $r_{1}<p$. Let $\delta$ be such that $1 / p<\delta<1 / r_{1}$ and let $f$ be as in (40). Since $\delta>1 / p$ we have, for any $M_{1}$,

$$
\left(\sum_{k} 2^{R k s p}\left|\Lambda_{R k} f(x)\right|^{p}\right)^{1 / p} \leq C_{M_{1}} \begin{cases}1 & \text { if }-N \leq x \leq 2 N \\ (1+|x|)^{-M_{1}} & \text { otherwise }\end{cases}
$$


This gives

$$
\left\|\left(\sum_{k} 2^{R k s p}\left|\Lambda_{R k} f\right|^{p}\right)^{1 / p}\right\|_{p, r_{0}} \lesssim N^{1 / p}, \quad r_{0}>0 .
$$

On the other hand we claim that

$$
2^{R k s}\left\|\Lambda_{R k} f\right\|_{p, r_{1}} \gtrsim(\log N)^{-\delta+1 / r_{1}}, \quad 5 N / 4 \leq k \leq 7 N / 4 .
$$

Let $\mathcal{V}_{\varepsilon, k, j}=\cup_{2^{j-1} \leq k-l \leq 2^{j}} B\left(R l e_{1}, \varepsilon\right)$, for $j$ with $0<2^{j} \leq N / 4$. We have

$$
\begin{aligned}
& 2^{R k s}\left|\Lambda_{R k} f(x)\right|=\left|\sum_{l=0}^{4 N}(1+R|k-l|)^{-1 / p} \log (2+R|k-l|)^{-\delta} \eta\left(x-R l e_{1}\right)\right| \\
& \geq c_{1} I_{k}(x)-C_{1} I I_{k}(x), \quad \text { with } \\
& I_{k}(x)=\sum_{0<2^{j}<N / 4}\left(1+R 2^{j}\right)^{-1 / p} \log \left(2+R 2^{j}\right)^{-\delta} \mathbb{1}_{\mathcal{V}_{\varepsilon, k, j}}(x), \\
& I I_{k}(x)=\sum_{\substack{0 \leq l \leq 4 N \\
\left|R l e_{1}-x\right| \geq R / 2}} \frac{\log (2+R|k-l|)^{-\delta}}{(1+R|k-l|)^{1 / p}} R^{-N_{1}}\left|x-R l e_{1}\right|^{-N_{1}} .
\end{aligned}
$$

It is immediate that $\left\|I I_{k}\right\|_{p} \lesssim R^{-N_{1}}$, for any $N_{1}$, and by interpolation also $\left\|I I_{k}\right\|_{p, r_{1}} \lesssim R^{-N_{1}}$.

Notice that meas $\left(\mathcal{V}_{\varepsilon, k, j}\right) \geq c \varepsilon^{d} 2^{j}$. For the rearrangement of $I_{k}$ we have

$$
I_{k}^{*}(t) \geq c \sum_{0 \leq 2^{j} \leq N / 8} 2^{-j / p} R^{-1 / p}\left[\log \left(R 2^{j}\right)\right]^{-\delta}(t) \mathbb{1}_{\left[0, c \varepsilon^{d} 2^{j}\right]}(t)
$$

and thus

$$
\begin{aligned}
\left\|I_{k}\right\|_{p, r_{1}} & \geq\left(\sum_{0<2^{j}<N / 8} \int_{c \varepsilon 2^{j-1}}^{c \varepsilon 2^{j}}\left[\left(R 2^{j}\right)^{-1 / p}\left(\log \left(R 2^{j}\right)\right)^{-\delta}\right]^{r_{1}} t^{r_{1} / p} \frac{d t}{t}\right)^{1 / r_{1}} \\
& \geq R^{-1 / p}(\log N)^{\frac{1-\delta r_{1}}{r_{1}}} .
\end{aligned}
$$

The two estimates for $\left\|I_{k}\right\|_{p, r_{1}}$ and $\left\|I I_{k}\right\|_{p, r_{1}}$ imply (44). Then also

$$
\|f\|_{B_{p}^{s}\left(L^{p, r_{1}}\right)} \gtrsim R^{-1 / p} N^{1 / p}(\log N)^{-\delta+1 / r_{1}}
$$

Comparing (43) and (45), and choosing $N$ large, we get a contradiction when $\delta<1 / r_{1}$. This means we must have $r_{1} \geq p$ in (39).

\section{Sequences of Vector-VAlued functions}

In order to prove the positive results in parts (iv)-(vi) of Theorems 1.1 and 1.2 we derive corresponding embeddings for spaces of sequences $\ell^{q}\left(L^{p, r}\right)$ and $L^{p, r}\left(\ell^{q}\right)$, for fixed $p, r$. 
Proposition 4.1. Let $0<p<\infty, 0<q_{0}, q_{1}, r_{0}, r_{1} \leq \infty$ and assume $q_{0} \leq \min \left\{p, q_{1}, r_{1}\right\}, r_{0} \leq r_{1}$. The embedding

$$
\ell^{q_{0}}\left(L^{p, r_{0}}\right) \hookrightarrow L^{p, r_{1}}\left(\ell^{q_{1}}\right)
$$

holds in each of the following three cases:

(i) $p \neq q_{1}$, (ii) $p=q_{1} \geq r_{0}$, (iii) $q_{0}<p=q_{1}<r_{0}$.

Proposition 4.2. Let $0<p<\infty, 0<q_{0}, q_{1}, r_{0}, r_{1} \leq \infty, r_{0} \leq r_{1}$ and $q_{1} \geq \max \left\{p, q_{0}, r_{0}\right\}$. The embedding

$$
L^{p, r_{0}}\left(\ell^{q_{0}}\right) \hookrightarrow \ell^{q_{1}}\left(L^{p, r_{1}}\right)
$$

holds in each of the following three cases:

(i) $p \neq q_{0}$, (ii) $p=q_{0} \leq r_{1}$, (iii) $r_{1}<q_{0}=p<q_{1}$.

Remark 4.3. In both Proposition 4.1 and Proposition 4.2 the assumptions (i), (ii), (iii) cannot be improved (unless one imposes very restrictive conditions on the underlying measure spaces). This follows from the examples for the spaces $B_{q}^{s}\left[L^{p, r}\right], F_{q}^{s}\left[L^{p, r}\right]$ discussed in $\$ 3$, although one can give less technical examples for the propositions.

We split the proof into several lemmata.

Lemma 4.4. Suppose $q \leq r \leq p$ or $q<p \leq r$. Then

$$
\ell^{q}\left(L^{p, r}\right) \hookrightarrow L^{p, r}\left(\ell^{q}\right)
$$

Proof. The asserted inequality is trivial when $p=q=r$. We may thus assume $p>q$. Then

$$
\|f\|_{L^{p, r}\left(\ell^{q}\right)}^{q}=\left\|\sum_{k}\left|f_{k}\right|^{q}\right\|_{L^{p / q, r / q}} \lesssim \sum_{k}\left\|\left|f_{k}\right|^{q}\right\|_{L^{p / q, r / q}}=\sum_{k}\left\|f_{k}\right\|_{L^{p, r}}^{q}
$$

Here we have used the triangle inequality in (18), for the space $L^{p / q, r / q}$, and twice the formula (15).

Lemma 4.5. Suppose that either $p \leq r \leq q$ or $r \leq p<q$. Then

$$
L^{p, r}\left(\ell^{q}\right) \hookrightarrow \ell^{q}\left(L^{p, r}\right)
$$

Proof. We first consider the case $r<\infty$ and argue by duality. Recall that if $A$ is a Banach space, $A^{\prime}$ its dual and $1<u<\infty$ then the dual of $\ell^{u}(A)$ is $\ell^{u^{\prime}}\left(A^{\prime}\right)$, with the natural pairing. Let $a<\min \{p, q, r\}$ and set $(P, Q, R)=$ $(p / a, q / a, r / a)$ so that $1<P, Q, R<\infty$. Since for $1<P, R<\infty$ the dual 
of $L^{P, R}$ is $L^{P^{\prime}, R^{\prime}}$ we see that

$$
\begin{aligned}
& \|f\|_{\ell^{q}\left(L^{p, r}\right)}^{a}=\left(\sum_{k}\left\|f_{k}\right\|_{L^{p, r}}^{q}\right)^{a / q} \\
& =\left(\sum_{k}\left\|\left|f_{k}\right|^{a}\right\|_{L^{P, Q}}^{Q}\right)^{1 / Q} \leq\left(\sum_{k}\left\|\left|f_{k}\right|^{a}\right\|_{L^{P, Q}}^{Q}\right)^{1 / Q} \\
& \lesssim \sup \left\{\sum_{k} \int\left|f_{k}(x)\right|^{a} g_{k}(x) d \mu(x):\|g\|_{\ell^{Q^{\prime}}\left(L^{P^{\prime}, R^{\prime}}\right)} \leq 1\right\}
\end{aligned}
$$

where the implicit constants depend on $p, q, r$. Now, let $\|g\|_{\ell Q^{\prime}\left(L^{P^{\prime}, R^{\prime}}\right)} \leq 1$. Then

$$
\begin{aligned}
& \sum_{k} \int\left|f_{k}(x)\right|^{a}\left|g_{k}(x)\right| d \mu \leq \int\left(\sum_{k}\left|f_{k}(x)\right|^{a Q}\right)^{1 / Q}\left(\sum_{k}\left|g_{k}(x)\right|^{Q^{\prime}}\right)^{1 / Q^{\prime}} d \mu \\
& \lesssim\left\|\left(\sum_{k}\left|f_{k}\right|^{a Q}\right)^{1 / Q}\right\|\left\|_{L^{P, R}}\right\|\left(\sum_{k}\left|g_{k}\right|^{Q^{\prime}}\right)^{1 / Q^{\prime}} \|_{L^{P^{\prime}, R^{\prime}}} \\
& \lesssim\left\|\left(\sum_{k}\left|f_{k}\right|^{a Q}\right)^{1 / a Q}\right\|_{L^{a P, a R}}^{a}\|g\|_{\ell Q^{\prime}\left(L^{P^{\prime}, R^{\prime}}\right)} \lesssim\|f\|_{L^{p, r(\ell q)}}^{a} ;
\end{aligned}
$$

here we have used for the second to last inequality that

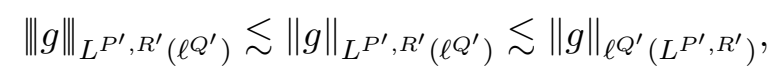

by Lemma 4.4 since $Q^{\prime} \leq R^{\prime} \leq P^{\prime}$ or $Q^{\prime}<P^{\prime} \leq R^{\prime}$. This completes the proof for $r<\infty$.

Next assume $r=\infty$, then also $q=\infty$. Clearly we have for any fixed $k_{0}$

$$
\mu\left(\left\{x:\left|f_{k_{0}}(x)\right|>\alpha\right\}\right) \leq \mu\left(\left\{x: \sup _{k}\left|f_{k}(x)\right|>\alpha\right\}\right) .
$$

Hence $\sup _{k} \sup _{\alpha>0} \alpha\left[\mu_{f_{k}}(\alpha)\right]^{1 / p} \leq \sup _{\alpha>0} \alpha\left[\mu_{\sup _{k}\left|f_{k}\right|}(\alpha)\right]^{1 / p}$ which yields the case $r=q=\infty$.

Next we state some weaker embedding properties for the case $p<q \leq r$.

Lemma 4.6. (i) Let $p<q \leq r$ or $p=q=r$. Then

$$
\ell^{p}\left(L^{p, r}\right) \hookrightarrow L^{p, r}\left(\ell^{q}\right)
$$

(ii) Let $v<p \leq r$. Then

$$
\ell^{v}\left(L^{p, r}\right) \hookrightarrow L^{p, r}\left(\ell^{p}\right)
$$

Proof. The statement is trivial for $q=r=p$. Let $p<q \leq r$. We use the modified $p / q$-triangle inequality in $L^{p / q, s}$ for $s=r / q \geq 1$, as in (20), and 
estimate

$$
\begin{aligned}
& \left\|\left(\sum_{k}\left|f_{k}\right|^{q}\right)^{1 / q}\right\|_{L^{p, r}}=\left\|\sum_{k}\left|f_{k}\right|^{q}\right\|_{L^{p / q, r / q}}^{1 / q} \\
& \lesssim\left(\left(\sum_{k}\left\|\left|f_{k}\right|^{q}\right\|_{L^{p / q, r / q}}^{p / q}\right)^{q / p}\right)^{1 / q}=\left(\sum_{k}\left\|f_{k}\right\|_{L^{p, r}}^{p}\right)^{1 / p} .
\end{aligned}
$$

For (ii) we use the embedding $\ell^{v} \subset \ell^{p}$ and then the triangle inequality in $L^{p / v, r / v}$ (cf. (18)) to obtain

$$
\begin{aligned}
& \left\|\left(\sum_{k}\left|f_{k}\right|^{p}\right)^{1 / p}\right\|_{L^{p, r}} \leq\left\|\left(\sum_{k}\left|f_{k}\right|^{v}\right)^{1 / v}\right\|_{L^{p, r}} \\
& \leq\left\|\sum_{k}\left|f_{k}\right|^{v}\right\|_{L^{p / v, r / v}}^{1 / v} \lesssim\left(\sum_{k}\left\|\left|f_{k}\right|^{v}\right\|_{L^{p / v, r / v}}\right)^{1 / v} \lesssim\left(\sum_{k}\left\|f_{k}\right\|_{L^{p, r}}^{v}\right)^{1 / v} .
\end{aligned}
$$

Lemma 4.7. (i) Let $0<r \leq q<p$ or $r=p=q$. Then

$$
L^{p, r}\left(\ell^{q}\right) \hookrightarrow \ell^{p}\left(L^{p, r}\right)
$$

(ii) Let $0<r \leq p<w$. Then

$$
L^{p, r}\left(\ell^{p}\right) \hookrightarrow \ell^{w}\left(L^{p, r}\right) .
$$

Proof. The statement is trivial for $r=p=q$. If $0<r \leq q<p<\infty$, set $(P, Q, R)=(p / a, q / a, r / a)$ for some $a<\min \{p, q, r\}$ and argue by duality exactly as in the proof of Lemma 4.5, basing the argument on Lemma 4.6.

Proof of Proposition 4.1. Let $q_{0} \leq \min \left\{p, q_{1}, r_{1}\right\}$. We distinguish the three cases according to whether $p, q_{1}$, or $r_{1}$ is the smallest exponent.

Case 1: $q_{0} \leq q_{1} \leq \min \left\{p, r_{1}\right\}$. If either $q_{0} \leq r_{1} \leq p$ or $q_{0}<p \leq r_{1}$ then $\ell^{q_{0}}\left(L^{p, r_{1}}\right) \hookrightarrow L^{p, r_{1}}\left(\ell^{q_{0}}\right)$ by Lemma 4.4 and hence

$$
\ell^{q_{0}}\left(L^{p, r_{0}}\right) \hookrightarrow \ell^{q_{0}}\left(L^{p, r_{1}}\right) \hookrightarrow L^{p, r_{1}}\left(\ell^{q_{0}}\right) \hookrightarrow L^{p, r_{1}}\left(\ell^{q_{1}}\right) .
$$

In the remaining subcase we have $q_{0}=q_{1}=p \leq r_{1}$ and by assumption of the proposition we also have $r_{0} \leq p$. Thus $\ell^{p}\left(L^{p, r_{0}}\right) \hookrightarrow \ell^{p}\left(L^{p}\right)=L^{p}\left(\ell^{p}\right) \hookrightarrow$ $L^{p, r_{1}}\left(\ell^{p}\right)$.

Case 2: $q_{0} \leq r_{1} \leq \min \left\{p, q_{1}\right\}$. Note that $\ell^{r_{1}}\left(L^{p, r_{1}}\right) \hookrightarrow L^{p, r_{1}}\left(\ell^{r_{1}}\right)$ for $r_{1} \leq p$, again by Lemma 4.4. Thus we obtain

$$
\ell^{q_{0}}\left(L^{p, r_{0}}\right) \hookrightarrow \ell^{r_{1}}\left(L^{p, r_{1}}\right) \hookrightarrow L^{p, r_{1}}\left(\ell^{r_{1}}\right) \hookrightarrow L^{p, r_{1}}\left(\ell^{q_{1}}\right) .
$$

In the third case $q_{0} \leq p \leq \min \left\{q_{1}, r_{1}\right\}$. The embedding is trivial when $p=q_{1}=r_{1}$. We distinguish three remaining subcases.

Case 3-1: $p<q_{1} \leq r_{1}$. We apply Lemma 4.6, (i), to get

$$
\ell^{q_{0}}\left(L^{p, r_{0}}\right) \hookrightarrow \ell^{p}\left(L^{p, r_{1}}\right) \hookrightarrow L^{p, r_{1}}\left(\ell^{q_{1}}\right) .
$$

Case 3-2: $q_{0} \leq p=q_{1}<r_{1}$. If $q_{0}<p$ then by part (ii) of Lemma 4.6.

$$
\ell^{q_{0}}\left(L^{p, r_{0}}\right) \hookrightarrow \ell^{q_{0}}\left(L^{p, r_{1}}\right) \hookrightarrow L^{p, r_{1}}\left(\ell^{p}\right) .
$$


If $q_{0}=p$ then we have by assumption also $r_{0} \leq p$ and therefore $\ell^{p}\left(L^{p, r_{0}}\right) \hookrightarrow$ $\ell^{p}\left(L^{p}\right)=L^{p}\left(\ell^{p}\right) \hookrightarrow L^{p, r_{1}}\left(\ell^{p}\right)$.

Case 3-3: $q_{0} \leq p \leq r_{1}<q_{1}$. Now observe that $\ell^{p}\left(L^{p, r_{1}}\right) \hookrightarrow L^{p, r_{1}}\left(\ell^{r_{1}}\right)$ by Lemma 4.6, (i). Hence

$$
\ell^{q_{0}}\left(L^{p, r_{0}}\right) \hookrightarrow \ell^{p}\left(L^{p, r_{1}}\right) \hookrightarrow L^{p, r_{1}}\left(\ell^{r_{1}}\right) \hookrightarrow L^{p, r_{1}}\left(\ell^{q_{1}}\right) .
$$

Proof of Proposition 4.2. The proof is 'dual' to the proof of Proposition 4.1. Formally the proof goes by reversing the arrows in the proof of Proposition 4.1 and replacing the subscripts $(0,1)$ by $(1,0)$. We now use Lemma 4.5 and Lemma 4.7 in place of Lemma 4.4 and Lemma 4.6. We run through the cases:

Case 1': $q_{1} \geq q_{0} \geq \max \left\{p, r_{0}\right\}$. If $p \leq r_{0} \leq q_{1}$, or $r_{0} \leq p<q_{1}$ and the second of the following embeddings holds by Lemma 4.5.

$$
L^{p, r_{0}}\left(\ell^{q_{0}}\right) \hookrightarrow L^{p, r_{0}}\left(\ell^{q_{1}}\right) \hookrightarrow \ell^{q_{1}}\left(L^{p, r_{0}}\right) \hookrightarrow \ell^{q_{1}}\left(L^{p, r_{1}}\right) .
$$

If $r_{0} \leq p=q_{0}=q_{1}$ then also by assumption $r_{1} \geq p$ and hence $L^{p, r_{0}}\left(\ell^{q_{0}}\right) \hookrightarrow$ $L^{p}\left(\ell^{p}\right)=\ell^{p}\left(L^{p}\right) \hookrightarrow \ell^{p}\left(L^{p, r_{1}}\right)$.

Case 2': $q_{1} \geq r_{0} \geq \max \left\{p, q_{0}\right\}$. First observe that Lemma 4.5 also implies $L^{p, r_{0}}\left(\ell^{r_{0}}\right) \hookrightarrow \ell^{r_{0}}\left(L^{p, r_{0}}\right)$ for $p \geq r_{0}$. Hence

$$
L^{p, r_{0}}\left(\ell^{q_{0}}\right) \hookrightarrow L^{p, r_{0}}\left(\ell^{r_{0}}\right) \hookrightarrow \ell^{r_{0}}\left(L^{p, r_{0}}\right) \hookrightarrow \ell^{q_{1}}\left(L^{p, r_{1}}\right) .
$$

The third case $q_{1} \geq p \geq \max \left\{q_{0}, r_{0}\right\}$ is again split into three subcases (ignoring the trivial case $p=q_{0}=r_{0}$ ).

Case 3-1': $p>q_{0} \geq r_{0}$. We apply Lemma 4.7 to obtain

$$
L^{p, r_{0}}\left(\ell^{q_{0}}\right) \hookrightarrow \ell^{p}\left(L^{p, r_{0}}\right) \hookrightarrow \ell^{q_{1}}\left(L^{p, r_{1}}\right) .
$$

Case 3-2': $q_{1} \geq p=q_{0}>r_{1}$. If $q_{1}>p$ we get by part (ii) of Lemma 4.7.

$$
L^{p, r_{0}}\left(\ell^{p}\right) \hookrightarrow \ell^{q_{1}}\left(L^{p, r_{0}}\right) \hookrightarrow \ell^{q_{1}}\left(L^{p, r_{1}}\right) .
$$

If $q_{1}=p$ then by assumption $r_{1} \geq p$ and therefore $L^{p}\left(\ell^{r_{0}}\right) \hookrightarrow L^{p}\left(\ell^{p}\right)=$ $\ell^{p}\left(L^{p}\right) \hookrightarrow \ell^{p}\left(L^{p, r_{1}}\right)$.

Case 3-3': $q_{1} \geq p \geq r_{0}>q_{0}$. We use that $L^{p, r_{0}}\left(\ell^{r_{0}}\right) \hookrightarrow \ell^{p}\left(L^{p, r_{0}}\right)$, by Lemma 4.7. Hence

$$
L^{p, r_{0}}\left(\ell^{q_{0}}\right) \hookrightarrow L^{p, r_{0}}\left(\ell^{r_{0}}\right) \hookrightarrow \ell^{p}\left(L^{p, r_{0}}\right) \hookrightarrow \ell^{q_{1}}\left(L^{p, r_{1}}\right) .
$$

We now get the statements (iv)-(vi) in Theorems 1.1 and 1.2 .

Corollary 4.8. Let $0<p<\infty, 0<q_{0}, q_{1}, r_{0}, r_{1} \leq \infty$.

(i) Suppose that either $p \neq q_{1}$ or that $p=q_{1} \geq r_{0}$. Then the embedding

$$
B_{q_{0}}^{s}\left[L^{p, r_{0}}\right] \hookrightarrow F_{q_{1}}^{s}\left[L^{p, r_{1}}\right]
$$

holds if and only if $q_{0} \leq \min \left\{p, q_{1}, r_{1}\right\}$ and $r_{0} \leq r_{1}$.

(ii) Let $r_{0}>p$. Then the embedding

$$
B_{q_{0}}^{s}\left[L^{p, r_{0}}\right] \hookrightarrow F_{p}^{s}\left[L^{p, r_{1}}\right]
$$

holds if and only if $q_{0}<p$ and $r_{0} \leq r_{1}$. 
Corollary 4.9. Let $0<p<\infty, 0<q_{0}, q_{1}, r_{0}, r_{1} \leq \infty$.

(i) Suppose either that $p \neq q_{0}$ or that $p=q_{0} \leq r_{1}$. Then the embedding

$$
F_{q_{0}}^{s}\left[L^{p, r_{0}}\right] \hookrightarrow B_{q_{1}}^{s}\left[L^{p, r_{1}}\right]
$$

holds if and only if $q_{1} \geq \max \left\{p, q_{0}, r_{0}\right\}$ and $r_{0} \leq r_{1}$.

(ii) Let $r_{1}<p$. Then the embedding

$$
F_{p}^{s}\left[L^{p, r_{0}}\right] \hookrightarrow B_{q_{1}}^{s}\left[L^{p, r_{1}}\right]
$$

holds if and only if $q_{1}>p$ and $r_{0} \leq r_{1}$.

Proof of Corollary 4.8 and Corollary 4.9 . The positive results follow immediately from the corresponding results in Propositions 4.1 and 4.2 when applied to $\left\{f_{k}\right\}_{k=0}^{\infty}$ with $f_{k}=2^{k s} \Lambda_{k} f$. The necessity of the conditions was proved in $\$ 3$.

\section{Embeddings of JaWerth-Franke type}

Jawerth's and Franke's versions of the Sobolev embedding theorem were reproved by Vybíral 32 using sequence spaces which are discrete variants of Besov and Triebel-Lizorkin spaces. The proofs are inspired by [32] For reasons of brevity and preference we choose not to introduce sequence spaces in the Lorentz category.

Preliminary considerations. We first need a straightforward Lorentz space version of Peetre's maximal theorem.

Lemma 5.1. Let $f_{k} \in \mathcal{S}^{\prime}$ be such that $\widehat{f}_{k}$ is supported in $\left\{\xi:|\xi| \leq 2^{k}\right\}$. Let

$$
\mathfrak{M}_{k} f_{k}(x)=\sup _{|h| \leq d 2^{-k}}\left|f_{k}(x+h)\right| \text {. }
$$

Then for $0<p<\infty, 0<q, r \leq \infty$,

$$
\left\|\left\{\mathfrak{M}_{k} f_{k}\right\}\right\|_{L^{p, r}\left(\ell^{q}\right)} \lesssim C_{p, q, r}\left\|\left\{f_{k}\right\}\right\|_{L^{p, r}\left(\ell^{q}\right)} .
$$

Proof. Let $M_{H L}$ be the Hardy-Littlewood maximal operator. We have

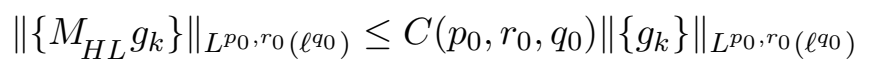

for $1<p_{0}, r_{0}, q_{0}<\infty$. The version for $p_{0}=r_{0}$ was proved by Fefferman and Stein [6] and the general version follows by real interpolation.

From [17] we have the inequality

$$
\mathfrak{M}_{k} f_{k}(x) \leq C_{\rho}\left(M_{H L}\left(\left|f_{k}\right|^{\rho}\right)(x)\right)^{1 / \rho}
$$

for all $\rho>0$. We choose $\rho<\min \{p, q, r\}$, and apply (47) with $\left(p_{0}, r_{0}, q_{0}\right)=$ $(p / \rho, r / \rho, q / \rho)$. Then

$$
\begin{aligned}
& \left\|\left\{\mathfrak{M}_{k} f_{k}\right\}\right\|_{L^{p, r}\left(\ell^{q}\right)} \lesssim\left\|\left\{\left(M_{H L}\left(\left|f_{k}\right|^{\rho}\right)\right)^{1 / \rho}\right\}\right\|_{L^{p, r}\left(\ell^{q}\right)} \\
& =\left\|\left\{M_{H L}\left(\left|f_{k}\right|^{\rho}\right)\right\}\right\|_{L^{\frac{p}{\rho}, \frac{r}{\rho}\left(\ell^{\frac{q}{\rho}}\right)}}^{1 / \rho} \lesssim\left\|\left\{\left|f_{k}\right|^{\rho}\right\}\right\|_{L^{\frac{p}{\rho}, \frac{r}{\rho}\left(\ell^{\frac{q}{\rho}}\right)}}^{1 / \rho}=\left\|\left\{f_{k}\right\}\right\|_{L^{p, r}\left(\ell^{q}\right)} .
\end{aligned}
$$


Theorem 5.2. Suppose $0<d\left(1 / p_{0}-1 / p_{1}\right)=s_{0}-s_{1}, 0<q_{0}, q_{1}, r_{0}, r_{1} \leq \infty$. Then the embedding $F_{q_{0}}^{s_{0}}\left[L^{p_{0}, r_{0}}\right] \hookrightarrow B_{q_{1}}^{s_{1}}\left[L^{p_{1}, r_{1}}\right]$ holds if and only if $r_{0} \leq q_{1}$.

Proof. The necessity of the condition $r_{0} \leq q_{1}$ has been established in 33.5 .

Let $Q_{k}(x)$ be the unique dyadic cube of sidelength $2^{-k}$ which contains $x$, (the sides being half open intervals). Set

$$
\begin{aligned}
& g_{k}(x)=2^{k s_{0}} \sup _{y \in Q_{k}(x)} \Lambda_{k} f(y), \\
& G(x)=\sup _{k} 2^{k s_{0}} \mathfrak{M}_{k} \Lambda_{k} f(x) .
\end{aligned}
$$

Clearly

$$
g_{k}(x) \leq 2^{k s_{0}} \mathfrak{M}_{k} \Lambda_{k} f(x) \leq G(x)
$$

and therefore $g_{k}^{*}(t) \leq G^{*}(t)$.

Since $g_{k}$ is constant on the dyadic cubes of sidelength $2^{-k}$ we see that $g_{k}^{*}$ is constant on dyadic intervals of length $2^{-k d}$. In particular

$$
\begin{aligned}
\left\|g_{k}\right\|_{p_{1}, r_{1}} & =\left(\frac{r_{1}}{p_{1}} \int_{0}^{\infty} t^{r_{1} / p} g_{k}^{*}(t)^{r_{1}} \frac{d t}{t}\right)^{1 / r_{1}} \\
& \approx\left(\sum_{n=1}^{\infty}\left[\left(2^{-k d} n\right)^{1 / p_{1}} g_{k}^{*}\left(2^{-k d}(n-1)\right)\right]^{r_{1}} \frac{2^{-k d}}{2^{-k d} n}\right)^{1 / r_{1}}
\end{aligned}
$$

We now begin with the proof of the sufficiency of the condition $q_{1} \geq r_{0}$. Since the $B_{q}^{s}\left(L^{p, r}\right), F_{q}^{s}\left(L^{p, r}\right)$ norms increase when $r$ decreases or when $q$ decreases, it suffices to consider the case $q_{1}=r_{0}=: \rho$ and to prove for $0<\rho \leq \infty$ and $r_{1}<\rho$

$$
F_{\infty}^{s_{0}}\left[L^{p_{0}, \rho}\right] \hookrightarrow B_{\rho}^{s_{1}}\left[L^{p_{1}, r_{1}}\right]
$$

We write the proof for $\rho<\infty$ but this is not essential as the case $\rho=\infty$ will only require notational changes.

Now

$$
\begin{aligned}
\|f\|_{B_{\rho}^{s_{1}}\left(L^{\left.p_{1}, r_{1}\right)}\right.} & =\left(\sum_{k=0}^{\infty} 2^{k s_{1} \rho}\left\|\Lambda_{k} f\right\|_{p_{1}, r_{1}}^{\rho}\right)^{1 / \rho} \\
& =\left(\sum_{k=0}^{\infty}\left\|2^{k s_{0}} \Lambda_{k} f\right\|_{p_{1}, r_{1}}^{\rho} 2^{-k d\left(\frac{1}{p_{0}}-\frac{1}{p_{1}}\right) \rho}\right)^{1 / \rho}
\end{aligned}
$$


Here we have used the relation $d / p_{0}-d / p_{1}=s_{0}-s_{1}$. The last displayed expression is dominated by

$$
\begin{aligned}
& \left(\sum_{k=0}^{\infty} 2^{-k d\left(\frac{1}{p_{0}}-\frac{1}{p_{1}}\right) \rho}\left\|g_{k}\right\|_{p_{1}, r_{1}}^{\rho}\right)^{1 / \rho} \\
& \lesssim\left(\sum_{k=0}^{\infty} 2^{-k d\left(\frac{1}{p_{0}}-\frac{1}{p_{1}}\right) \rho}\left[\sum_{n=1}^{\infty}\left(2^{-k d} n\right)^{r_{1} / p_{1}} g_{k}^{*}\left(2^{-k d}(n-1)\right)^{r_{1}} \frac{1}{n}\right]^{\rho / r_{1}}\right)^{1 / \rho} \\
& \leq\left(\sum_{k=0}^{\infty}\left(\sum_{n=1}^{\infty} n^{r_{1}\left(\frac{1}{p_{1}}-\frac{1}{p_{0}}\right)-1} G^{*}\left(2^{-k d}(n-1)\right)^{r_{1}}\left(2^{-k d} n\right)^{r_{1} / p_{0}}\right)^{\rho / r_{1}}\right)^{1 / \rho}
\end{aligned}
$$

The last expression is comparable to

$$
\begin{aligned}
& \left(\sum_{k=0}^{\infty}\left(\sum_{j=0}^{\infty} 2^{j r_{1}\left(1 / p_{1}-1 / p_{0}\right)} G^{*}\left(2^{-k d+j-1}\right)^{r_{1}}\left(2^{-k d+j}\right)^{r_{1} / p_{0}}\right)^{\rho / r_{1}}\right)^{1 / \rho} \\
& \lesssim\left(\sum_{j=0}^{\infty} 2^{j r_{1}\left(1 / p_{1}-1 / p_{0}\right)}\left(\sum_{k=0}^{\infty} G^{*}\left(2^{-k d+j-1}\right)^{\rho}\left(2^{-k d+j}\right)^{\rho / p_{0}}\right)^{r_{1} / \rho}\right)^{1 / r_{1}} \\
& \lesssim\left(\sum_{j=0}^{\infty} 2^{j r_{1}\left(1 / p_{1}-1 / p_{0}\right)}\left(\sum_{k=0}^{\infty} \int_{2^{-k d+j-1}}^{2^{-k d+j}} t^{\rho / p_{0}} G^{*}(t)^{\rho} \frac{d t}{t}\right)^{r_{1} / \rho}\right)^{1 / r_{1}}
\end{aligned}
$$

Here we have used the triangle inequality in $L^{\rho / r_{1}}$ (as $\left.\rho / r_{1} \geq 1\right)$. Now for fixed $j$ the intervals $\left[2^{-k d+j-1}, 2^{-k d+j}\right]$ have disjoint interior and therefore the last expression is dominated by

$$
\begin{aligned}
& \left(\sum_{j=0}^{\infty} 2^{j r_{1}\left(1 / p_{1}-1 / p_{0}\right)}\|G\|_{p_{0}, \rho}^{r_{1}}\right)^{1 / r_{1}} \\
& \lesssim\|G\|_{p_{0}, \rho} \lesssim\left\|\sup _{k} 2^{k s_{0}} \mid \Lambda_{k} f\right\|_{p_{0}, \rho}=\|f\|_{F_{\infty}^{s_{0}}\left[L^{p_{0}, \rho}\right]}
\end{aligned}
$$

and (48) is proved.

Theorem 5.3. Suppose $0<d\left(1 / p_{0}-1 / p_{1}\right)=s_{0}-s_{1}, 0<q_{0}, q_{1}, r_{0}, r_{1} \leq \infty$. Then the embedding $B_{q_{0}}^{s_{0}}\left[L^{p_{0}, r_{0}}\right] \hookrightarrow F_{q_{1}}^{s_{1}}\left[L^{p_{1}, r_{1}}\right]$ holds if and only if $q_{0} \leq r_{1}$.

Proof. For the necessity of the condition $q_{0} \leq r_{1}$ see 33.5 . It now suffices to prove for any $q>0$ and for $0<\rho \leq \infty$,

$$
B_{\rho}^{s_{0}}\left[L^{p_{0}, \infty}\right] \hookrightarrow F_{q}^{s_{1}}\left[L^{p_{1}, \rho}\right] .
$$

Assume that $f \in B_{\rho}^{s_{0}}\left[L^{p_{0}, \infty}\right]$. Define

$$
h_{k}(x)=2^{k s_{1}} \sum_{Q \in \mathfrak{Q}_{k}} \mathbb{1}_{Q}(x) \inf _{Q} \mathfrak{M}_{k}\left(\Lambda_{k} f\right)
$$


where $\mathfrak{Q}_{k}$ denotes the grid of dyadic cubes with side length $2^{-k}$. Then

$$
\begin{aligned}
\|f\|_{F_{q}^{s_{1}}\left(L^{p_{1}, \rho}\right)} & =\left\|\sum_{k=0}^{\infty} 2^{k s_{1} q}\left|\Lambda_{k} f\right|^{q}\right\|_{p_{1} / q, \rho / q}^{1 / q} \leq\left\|\sum_{k=0}^{\infty} h_{k}^{q}\right\|_{p_{1} / q, \rho / q}^{1 / q} \\
& =\left(\sup _{\|g\|_{\left(p_{1} / q\right)^{\prime},(\rho / q)^{\prime}}=1} \sum_{k=0}^{\infty} \int h_{k}(x)^{q} g(x) d x\right)^{1 / q} .
\end{aligned}
$$

Note that the rearrangement function of $h_{k}$ is constant on the intervals $\left[2^{-k d}(n-1), 2^{-k d} n\right)$ for $n=1,2, \ldots$ Thus for fixed $k$

$$
\begin{aligned}
& \left|\int h_{k}(x)^{q} g(x) d x\right| \leq \int_{0}^{\infty}\left(h_{k}^{q}\right)^{*}(t) g^{*}(t) d t \\
& =\int_{0}^{\infty}\left(h_{k}^{*}(t)\right)^{q} g^{*}(t) d t=\sum_{n=1}^{\infty} h_{k}^{*}\left(2^{-k d}(n-1)\right) \int_{2^{-k d}(n-1)}^{2^{-k d} n} g^{*}(t) d t \\
& \leq \sum_{n=1}^{\infty}\left(h_{k}^{*}\left(2^{-k d}(n-1)\right)\right)^{q} 2^{-k d} g^{* *}\left(2^{-k d} n\right)
\end{aligned}
$$

We sum in $k$ and get

$$
\begin{aligned}
& \sum_{k=0}^{\infty} \sum_{n=1}^{\infty}\left(h_{k}^{*}\left(2^{-k d}(n-1)\right)\right)^{q} 2^{-k d} g^{* *}\left(2^{-k d} n\right) \\
& \lesssim \sum_{k=0}^{\infty} \sum_{l=0}^{\infty}\left(h_{k}^{*}\left(2^{l d-k d-1}\right)\right)^{q} 2^{l d-k d} g^{* *}\left(2^{l d-k d}\right) \\
& =\sum_{l=0}^{\infty} \sum_{k=0}^{\infty} 2^{(l d-k d) q / p_{0}} 2^{k\left(s_{0}-s_{1}\right) q} h_{k}^{*}\left(2^{l d-k d-1}\right)^{q} \times \\
& 2^{(l d-k d)\left(1-q / p_{0}\right)} g^{* *}\left(2^{l d-k d}\right) 2^{-k d\left(1 / p_{0}-1 / p_{1}\right) q}
\end{aligned}
$$

where we have used $s_{0}-s_{1}=d / p_{0}-d / p_{1}$. By Hölder's inequality the last displayed expression is dominated by

$$
\begin{aligned}
& \sum_{l=0}^{\infty}\left(\sum_{k=0}^{\infty} 2^{(l d-k d) \rho / p_{0}} 2^{k\left(s_{0}-s_{1}\right) \rho} h_{k}^{*}\left(2^{l d-k d-1}\right)^{\rho}\right)^{q / \rho} \times \\
& \left(\sum_{k=0}^{\infty}\left[2^{(l d-k d)\left(1-q / p_{0}\right)} g^{* *}\left(2^{l d-k d}\right) 2^{-k d\left(1 / p_{0}-1 / p_{1}\right) q}\right]^{(\rho / q)^{\prime}}\right)^{1-q / \rho} \\
& \lesssim\left(\sum_{k=0}^{\infty} 2^{k\left(s_{0}-s_{1}\right) \rho} \sup _{m \geq 0} 2^{(m d-k d-1) \rho / p_{0}} h_{k}^{*}\left(2^{m d-k d-1}\right)^{\rho}\right)^{q / \rho} \times \\
& \sum_{l=0}^{\infty}\left(\sum_{k=0}^{\infty}\left[2^{(l d-k d)\left(1-q / p_{0}\right)} g^{* *}\left(2^{l d-k d}\right) 2^{-k d\left(1 / p_{0}-1 / p_{1}\right) q}\right]^{(\rho / q)^{\prime}}\right)^{1-q / \rho} .
\end{aligned}
$$


Now we have

$$
\begin{aligned}
& \left(\sum_{k=0}^{\infty} 2^{k\left(s_{0}-s_{1}\right) \rho} \sup _{m \geq 0} 2^{(m d-k d-1) \rho / p_{0}} h_{k}^{*}\left(2^{m d-k d-1}\right)^{\rho}\right)^{q / \rho} \\
& \lesssim\left(\sum_{k=0}^{\infty} 2^{k s_{0} \rho}\left\|2^{-k s_{1}} h_{k}\right\|_{p_{0}, \infty}^{\rho}\right)^{q / \rho} \lesssim\left(\sum_{k=0}^{\infty} 2^{k s_{0} \rho}\left\|\mathfrak{M}_{k}\left(\Lambda_{k} f\right)\right\|_{p_{0}, \infty}^{\rho}\right)^{q / \rho} \\
& \lesssim\left(\sum_{k=0}^{\infty} 2^{k s_{0} \rho}\left\|\Lambda_{k} f\right\|_{p_{0}, \infty}^{\rho}\right)^{q / \rho}=\|f\|_{B_{\rho}^{s_{0}}\left[L^{p_{0}, \infty}\right]^{\cdot}}^{q}
\end{aligned}
$$

Finally we estimate, summing $\sum_{l=0}^{\infty} 2^{-l d\left(1 / p_{0}-1 / p_{1}\right) q} \lesssim 1$,

$$
\begin{aligned}
& \sum_{l=0}^{\infty}\left(\sum_{k=0}^{\infty}\left[2^{(l d-k d)\left(1-q / p_{0}\right)} g^{* *}\left(2^{l d-k d}\right) 2^{-k d\left(1 / p_{0}-1 / p_{1}\right) q}\right]^{(\rho / q)^{\prime}}\right)^{1-q / \rho} \\
& \lesssim \sup _{l \geq 0}\left(\sum_{k=0}^{\infty}\left[2^{(l d-k d)\left(1-q / p_{0}\right)} g^{* *}\left(2^{l d-k d}\right) 2^{(l d-k d)\left(1 / p_{0}-1 / p_{1}\right) q}\right]^{(\rho / q)^{\prime}}\right)^{1-q / \rho} \\
& =\sup _{l \geq 0}\left(\sum_{k=0}^{\infty}\left[2^{(k d-l d)\left(1-q / p_{1}\right)} g^{* *}\left(2^{l d-k d}\right)\right]^{(\rho / q)^{\prime}}\right)^{1-\rho / q} \lesssim\|g\|_{\left(p_{1} / q\right)^{\prime},(\rho / q)^{\prime}} \lesssim 1 .
\end{aligned}
$$

Going back to (50) we see that $\|f\|_{F_{q}^{s_{1}}\left[L^{p_{1}, \rho}\right]} \lesssim\|f\|_{B_{\rho}^{s_{0}}\left[L^{p_{0}, \infty}\right]}$ and the proof of (49) is complete.

\section{Conclusion of the proofs of Theorems 1.1, 1.2, 1.5, 1.6}

The necessity of all conditions was shown in $\oint 3$. The proofs of the embeddings in parts (iv)-(vi) of Theorem 1.1 and Theorem 1.2 were shown in 4 (see Corollaries 4.8 and 4.9). The embeddings in part (iii) of Theorem 1.1 and Theorem 1.2 are covered by Theorem 5.3 and Theorem 5.2 , respectively.

We consider part (ii) of Theorem 1.1. Let $s_{0}>s_{1}, r_{0} \leq r_{1}, p_{0}=p_{1}=p$. Let $\varepsilon>0$ such that $s_{0}-\varepsilon>s_{1}$, and let $v<\min \left\{q_{0}, p_{1}, q_{1}, r_{1}\right\}$. By parts (iv) or (v) of Theorem 1.1 we have $B_{v}^{s_{0}-\varepsilon}\left[L^{p, r_{0}}\right] \hookrightarrow F_{q_{1}}^{s_{0}-\varepsilon}\left[L^{p, r_{1}}\right]$ and (ii) follows if we combine this with the trivial embeddings $B_{q_{0}}^{s_{0}}\left[L^{p, r_{0}}\right] \hookrightarrow B_{v}^{s_{0}-\varepsilon}\left[L^{p, r_{0}}\right]$ and $F_{q_{1}}^{s_{0}-\varepsilon}\left[L^{p, r_{1}}\right] \hookrightarrow F_{q_{1}}^{s_{1}}\left[L^{p, r_{1}}\right]$.

The proof of part (ii) of Theorem 1.2 is similar. Moreover if we use part (iii) in Theorems 1.1 and 1.2 the proofs of part (i) in those theorems follows the same pattern as above.

Finally we consider Theorems 1.5 and 1.6. Part (iv) of these theorems are proved by using embeddings of $L^{p, r}$ spaces and of $\ell^{q}$ spaces.

To see part (iii) of Theorem 1.5. assume $q_{0} \leq q_{1}$ and let $\widetilde{p}$ and $\widetilde{s}$ be such that $p_{0}<\widetilde{p}<p_{1}, s_{1}<\widetilde{s}<s_{0}$ and $\widetilde{s}-s_{1}=d / \widetilde{p}-d / p_{1}$ and thus $s_{0}-\widetilde{s}=d / p_{0}-d / \widetilde{p}$. Pick $\widetilde{r}$ such that $q_{0} \leq \widetilde{r} \leq q_{1}$ and then

$$
B_{q_{0}}^{s_{0}}\left[L^{p_{0}, r_{0}}\right] \hookrightarrow F_{\widetilde{q}}^{\widetilde{s}}\left[L^{\widetilde{p}, \widetilde{r}}\right] \hookrightarrow B_{q_{1}}^{s_{1}}\left[L^{p_{1}, r_{1}}\right]
$$


for arbitrary $\widetilde{q}$, by Theorem 5.3 for the first embedding and Theorem 5.2 for the second.

To see part (iii) of Theorem 1.6 assume $r_{0} \leq r_{1}$. Pick $\widetilde{q}$ such that $r_{0} \leq$ $\widetilde{q} \leq r_{1}$ and then

$$
F_{q_{0}}^{s_{0}}\left[L^{p_{0}, r_{0}}\right] \hookrightarrow B_{\widetilde{q}}^{\widetilde{s}}\left[L^{\widetilde{p}, \widetilde{r}}\right] \hookrightarrow F_{q_{1}}^{s_{1}}\left[L^{p_{1}, r_{1}}\right]
$$

for arbitrary $\widetilde{r}$, by Theorem 5.2 for the first embedding and Theorem 5.3 for the second.

Given parts (iv), (iii) of Theorems 1.5 and 1.6 the parts (i), (ii) in the noncritical ranges can be obtained by the argument above.

\section{Appendix A. Remarks on Mikhlin-Hörmander multipliers}

Part (iii) of Theorems 1.1 and 1.2 (i.e. Theorems 5.3 and 5.2 ) can be applied to clarify the connection between certain sharp versions of the MikhlinHörmander multiplier theorem ([11]). Set $T_{m} f=\mathcal{F}^{-1}[m \widehat{f}]$. Let $\varphi$ be a nontrivial radial smooth functions which is compactly supported in $\mathbb{R}^{d} \backslash\{0\}$. We first recall the endpoint bound

$$
\left\|T_{m}\right\|_{L^{p} \rightarrow L^{p, 2}} \lesssim \sup _{t>0}\|\varphi m(t \cdot)\|_{B_{1}^{s}\left[L^{d / s}\right]}, \quad s=d(1 / p-1 / 2), 1<p \leq 2,
$$

which was proved by one of the authors in [18]. Moreover one gets $H^{1} \rightarrow$ $L^{1,2}$ boundedness under the condition $\sup _{t>0}\|\varphi m(t \cdot)\|_{B_{1}^{d / 2}\left[L^{2}\right]}<\infty$, see [19]. Note that (51) immediately implies that

$$
\left\|T_{m}\right\|_{L^{p} \rightarrow L^{p}} \lesssim \sup _{t>0}\|\varphi m(t \cdot)\|_{B_{1}^{s}\left[L^{d / s}\right]}, \quad d|1 / p-1 / 2|<s<d .
$$

Indeed, by the standard Sobolev imbedding theorem for Besov spaces we may assume that $s<d / 2$. Define $p_{0}$ by $d\left(1 / p_{0}-1 / 2\right)=s$, so that $1<p_{0}<$ $p<2$. Then (51) gives $L^{p_{0}} \rightarrow L^{p_{0}, 2}$ boundedness, and by the Marcinkiewicz interpolation theorem, and a subsequent duality argument we get (52a).

A recent result by Grafakos and Slavíková [9] states that for $1<p<\infty$

$$
\begin{aligned}
\left\|T_{m}\right\|_{L^{p} \rightarrow L^{p}} & \lesssim \sup _{t>0}\left\|(I-\Delta)^{s / 2}[\varphi m(t \cdot)]\right\|_{L^{d / s, 1}}, \\
& \approx \sup _{t>0}\|\varphi m(t \cdot)\|_{F_{2}^{s}\left(L^{d / s, 1}\right)}, \quad d|1 / p-1 / 2|<s<d,
\end{aligned}
$$

$c f$. (3). For fixed $s$ the relation between the norms on the right hand side in (52a) and (52b) is not immediately clear. The spaces $F_{2}^{s}\left[L^{d / s, 1}\right]$ and $B_{1}^{s}\left[L^{d / s}\right]$ are not comparable; we have $F_{2}^{s}\left[L^{d / s, 1}\right] \nsubseteq B_{1}^{s}\left[L^{d / s}\right]$ by 93.6 .2 , and we get $B_{1}^{s}\left[L^{d / s}\right] \nsubseteq F_{2}^{s}\left[L^{d / s, 1}\right]$ by the necessity of the condition $r_{0} \leq r_{1}$ in 3.3. However we do have the embeddings

$$
B_{1}^{s_{3}}\left[L^{d / s_{3}}\right] \hookrightarrow F_{q}^{s_{2}}\left[L^{d / s_{2}, 1}\right] \hookrightarrow B_{1}^{s_{1}}\left[L^{d / s_{1}}\right], \quad s_{1}<s_{2}<s_{3}, q>0 .
$$

The first inclusion in (53) follows from Theorem 1.1(iii) and the second from Theorem 1.2 (iii). Since both statements (52a), (52b) involve the same open 
$s$-interval we may apply (53) for $s_{1}>d|1 / p-1 / 2|$ and $q=2$ to see that they cover $L^{p}$ boundedness for exactly the same set of multiplier transformations.

Further directions and open problems.

A.1. Is there an endpoint inequality such as (51) in terms of localized $F_{2}^{s}\left[L^{d / s, 1}\right]$ spaces, when $s=d|1 / p-1 / 2|$ ?

A.2. It was proved in 20 that

$$
\left\|T_{m}\right\|_{L^{p} \rightarrow L^{p}} \lesssim \sup _{t>0}\|\varphi m(t \cdot)\|_{B_{1}^{d|1 / p-1 / 2|}\left[L^{q}\right]}
$$

for $1 / q>|1 / p-1 / 2|, 1<p<\infty$ (see also [1], [20] for corresponding results on Hardy spaces). In the first version of our paper posted as arXiv:1801.10570 in January 2018 we raised the question whether the space $B_{1}^{d|1 / p-1 / 2|}\left[L^{q}\right]$ in this result can be replaced with the Lorentz-Sobolev space $H_{(q, r)}^{d|1 / p-1 / 2|} \equiv F_{2}^{d|1 / p-1 / 2|}\left[L^{q, r}\right]$. A negative answer to this question was recently given by Slavíková [22. However the following question remains interesting.

A.2.1. Question. Let $1 / q>|1 / p-1 / 2|$. For which $u>1$, if any, can one

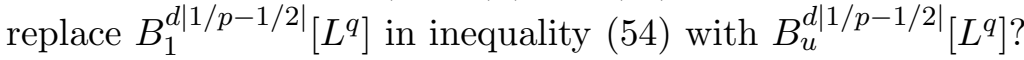

The parameter range $1<u \leq p$ seems of particular interest, as will be discussed in the following subsection.

A.3. Let $1 \leq p \leq 2$ and let $M^{p}$ be the usual Fourier multiplier space. We note the following obvious chain of inequalities

$$
\sup _{t>0}\left\|\mathcal{F}^{-1}[\varphi m(t \cdot)]\right\|_{L^{p}} \lesssim \sup _{t>0}\|\varphi m(t \cdot)\|_{M^{p}} \lesssim\|m\|_{M^{p}} .
$$

The corresponding inclusions for the spaces defined by the above norms are known to be strict. It is of interest to identify function spaces for which $\sup _{t>0}\left\|\mathcal{F}^{-1}[\varphi m(t \cdot)]\right\|_{L^{p}}$ is finite and the corresponding multiplier question is open. As an example one has the inequality

$$
\sup _{t>0}\left\|\mathcal{F}^{-1}[\varphi m(t \cdot)]\right\|_{L^{p}} \lesssim \sup _{t>0}\|\varphi m(t \cdot)\|_{B_{p}^{d(1 / p-1 / 2)}\left(L^{2}\right)}
$$

by using Bernstein's inequality. In view of the compact support of $\varphi$ we can replace $B_{p}^{d(1 / p-1 / 2)}\left(L^{2}\right)$ with $B_{p}^{d(1 / p-1 / 2)}\left(L^{q, r}\right)$ for $2<q<\infty, 0<r \leq \infty$.

We observe that a slight modification of the above mentioned example by Slavíková [22] exhibits a sequence of functions $g_{K}$ which is bounded in $F_{2}^{d(1 / p-1 / 2)}\left(L^{q, r}\right)$, with $2 \leq q<\infty, r>0$, for which $\sup _{t>0}\left\|\mathcal{F}^{-1}\left[\varphi g_{K}(t \cdot)\right]\right\|_{L^{p}}$ is unbounded. Indeed one may choose this sequence to be supported in $\{\xi: 1 / 4<|\xi|<2\}$.

To define $g_{K}$ let $\Psi$ be any nontrivial $C_{c}^{\infty}\left(\mathbb{R}^{d}\right)$ function supported on a ball of radius $1 / 10$ centered at the origin of $\mathbb{R}^{d}$. Let $K, N$ be large integers 
let $(k, N) \mapsto \nu(k, N)$ be an enumeration of $\mathbb{N}^{d} \times \mathbb{N}$ and denote by $r_{\nu}$ the Rademacher functions. Let, for $0 \leq s<\lfloor M+1\rfloor$ and $t \in[0,1]$,

$$
\begin{aligned}
& g_{s, K}(\xi, t)=\sum_{K / 2<N \leq K}\left(K 2^{N}\right)^{-s} h_{K, N}(\xi, t) \\
& \text { where } h_{K, N}(\xi, t)=\sum_{\substack{\kappa \in \mathbb{N}^{d}: \\
N<2^{-N}|\kappa|<N+1 / 2}} r_{\nu(k, N)}(t) \Psi\left(2^{N} K\left(\xi-2^{-N} K^{-1} \kappa\right)\right) .
\end{aligned}
$$

Then $\xi \mapsto g_{s}(\xi, t)$ is supported in the annulus $\mathcal{A}_{1}=\{1 / 4<|\xi| \leq 1\}$. The calculation in [22] (using Khinchine's inequality) shows that

$$
\left(\int_{0}^{1}\left\|\mathcal{F}^{-1}\left[g_{s, K}(\cdot, t)\right]\right\|_{p}^{p} d t\right)^{1 / p} \gtrsim_{s} K^{1 / p-1 / 2}, \text { when } s=d\left(\frac{1}{p}-\frac{1}{2}\right) .
$$

Moreover,

$$
\sup _{t \in[0,1]}\left\|g_{s, K}(\cdot, t)\right\|_{F_{2}^{s}\left(L^{q, r}\right)} \leq C(s), \quad 1<q<\infty, r>0,
$$

with polynomial growth in $s$. This can be verified by direct calculation as in [22]. Alternatively, one can immediately check this inequality for $r=q$ and $s=0,1,2, \ldots$ by using the standard Sobolev norms, and then apply an analytic interpolation argument to get the same statement for all $s \geq 0$. Fixing $s$, one can then apply real interpolation (with varying $q$ ) to get the statement for all $r>0, s \geq 0$.

In view of question A.2.1 it is instructive to replace $F_{2}^{s}\left(L^{q, r}\right)$ by $B_{u}^{s}\left(L^{q, r}\right)$ for some $q \geq 2, u \leq q$. We shall now impose the assumption that the generating function $\Psi$ satisfies $\int \Psi(y) P(y) d y=0$ for all polynomials of degree at most $M$, for some $M>s+2 d$. It is easy to check that the argument 22] for the lower bound (56) still goes through. We also have

$$
\sup _{t \in[0,1]}\left\|g_{s, K}(\cdot, t)\right\|_{B_{u}^{s}\left(L^{q, r}\right)} \lesssim K^{1 / u-1 / q}, \quad 2 \leq q<\infty, r>0, u \leq q .
$$

This is verified by using the Littlewood-Paley type characterization using compactly supported $\psi_{k}$ in (27), imposing the condition that $\psi_{k}$ for $k>0$ have a large number of vanishing moments, say more than $s+d+A$ for large $A$. One verifies that for $k \geq 0$

$$
\left\|\psi_{k} * h_{K, N}\right\|_{L^{q, r}} \lesssim K^{-1 / q} \min \left\{2^{N} K 2^{-k},\left(2^{N} K 2^{-k}\right)^{-1}\right\}^{-1-s},
$$

first for $q=r$ and then for general $r$ by real interpolation. This can be used to check (58).

Inequalities (56) and (58) show that the inequality

$$
\left\|\mathcal{F}^{-1} g\right\|_{L^{p}} \lesssim\|g\|_{B_{u}^{d(1 / p-1 / 2)}\left(L^{q, r}\right)}
$$

may fail for compactly supported $g$ when $u \leq q, 1 / u-1 / q<1 / p-1 / 2$, $r>0$. By (55) and its version with $B_{p}^{d(1 / p-1 / 2)}\left(L^{q, r}\right)$ for $q>2$ the range $1<u \leq p$ is of particular interest in question A.2.1. 


\section{Appendix B. On the Constant in the \\ TrIANGLe INEQUALITY FOR $L^{p, r}, p<1$}

In what follows we work with the quasinorm $\|\cdot\|_{p, r}$ on $L^{p, r}$ as defined in (9) or (10). The following result was referenced in 92 , together with an open question.

Proposition B.1. Let $0<p<1, p<r<\infty$. Then

$$
\left\|\sum_{k} f_{k}\right\|_{p, r} \leq C(p, r)\left(\sum_{k}\left\|f_{k}\right\|_{p, r}^{p}\right)^{1 / p}
$$

where

$$
C(p, r) \leq A^{1 / p}\left(\frac{1}{1-p}\right)^{1 / p-1 / r}\left(1+\frac{p}{r} \log \frac{1}{1-p}\right)^{1 / p-1 / r}
$$

and $A$ does not depend on $p$ and $r$.

We shall need the following lemma. It can be used to prove the inequality (11) when applied in combination with (10).

Lemma B.2. Let $g: \mathbb{R}^{+} \rightarrow \mathbb{R}$ be a Riemann integrable function and let $r<q$. Then for $0 \leq \alpha<\beta \leq \infty, 0<p<\infty$,

$$
\sup _{\alpha \leq \sigma \leq \beta} \sigma|g(\sigma)|^{1 / p} \leq\left(q \int_{\alpha}^{\beta} \sigma^{q}|g(\sigma)|^{q / p} \frac{d \sigma}{\sigma}\right)^{1 / q} \leq\left(r \int_{\alpha}^{\beta} \sigma^{r}|g(\sigma)|^{r / p} \frac{d \sigma}{\sigma}\right)^{1 / r} .
$$

Proof. We prove the second inequality, as the first one follows by letting $q \rightarrow \infty$. We may assume that $g$ is a nonnegative step function on $[\alpha, \beta]$, i.e. there is a partition $\alpha=b_{0}<b_{1}<\cdots<b_{N}=\beta$ so that $g(s)=c_{j}$ if $b_{j-1}<s<b_{j}$; here $c_{j} \geq 0$. The inequality then reduces to

$$
\left(\sum_{j=1}^{N} c_{j}^{q / p}\left(b_{j}^{q}-b_{j-1}^{q}\right)\right)^{1 / q} \leq\left(\sum_{j=1}^{N} c_{j}^{r / p}\left(b_{j}^{r}-b_{j-1}^{r}\right)\right)^{1 / r}
$$

We set $v_{j}=c_{j}^{r / p}, a_{j}=b_{j}^{r}$, and $s=q / r$ so that $s>1$, and see that the last inequality follows from

$$
\left(\sum_{j=1}^{N} v_{j}^{s}\left(a_{j}^{s}-a_{j-1}^{s}\right)\right)^{1 / s} \leq \sum_{j=1}^{N} v_{j}\left(a_{j}-a_{j-1}\right) .
$$

Since $s \geq 1$ we may (by the triangle inequality for the $s$-norms) replace $\left(a_{j}^{s}-a_{j-1}^{s}\right)$ on the left hand side of (61) with $\left(a_{j}-a_{j-1}\right)^{s}$, and (61) follows from $\|\cdot\|_{\ell^{s}} \leq\|\cdot\|_{\ell^{1}}$.

Proof of Proposition [B.1. The proof is based on ideas in [27], 25]. For given $\alpha>0$ we split $f_{k}=g_{k, \alpha}+b_{k, \alpha}$ where

$$
g_{k, \alpha}(x)= \begin{cases}f_{k}(x) & \text { if }\left|f_{k}(x)\right| \leq \alpha \\ 0 & \text { if }\left|f_{k}(x)\right|>\alpha\end{cases}
$$


and let $b_{k, \alpha}=f_{k}-g_{k, \alpha}$. Let

$$
E_{\alpha}=\left\{x: b_{k, \alpha}(x) \neq 0 \text { for some } k\right\} .
$$

Then

$$
\mu\left(\left\{x:\left|\sum_{k} f_{k}(x)\right|>\alpha\right\}\right) \leq \mu\left(E_{\alpha}\right)+\mu\left(\left\{x: \sum_{k}\left|g_{k, \alpha}(x)\right|>\alpha\right\}\right)
$$

and therefore

$$
\begin{aligned}
\left\|\sum_{k} f_{k}\right\|_{p, r}^{p} \leq( & \left.r \int_{0}^{\infty} \alpha^{r} \mu\left(E_{\alpha}\right)^{r / p} \frac{d \alpha}{\alpha}\right)^{p / r} \\
& +\left(r \int_{0}^{\infty} \alpha^{r}\left[\mu\left(\left\{x: \sum_{k}\left|g_{k, \alpha}(x)\right|>\alpha\right\}\right)\right]^{r / p} \frac{d \alpha}{\alpha}\right)^{p / r} .
\end{aligned}
$$

Now

$$
\mu\left(E_{\alpha}\right) \leq \sum_{k} \mu\left(\left\{x: b_{k, \alpha}(x) \neq 0\right\}\right) \leq \sum_{k} \mu_{f_{k}}(\alpha)
$$

and hence

$$
\begin{aligned}
& \left(r \int_{0}^{\infty} \alpha^{r} \mu\left(E_{\alpha}\right)^{r / p} \frac{d \alpha}{\alpha}\right)^{p / r} \leq\left(r \int_{0}^{\infty} \alpha^{r}\left(\sum_{k} \mu_{f_{k}}(\alpha)\right)^{r / p} \frac{d \alpha}{\alpha}\right)^{p / r} \\
& \leq \sum_{k}\left(r \int_{0}^{\infty} \alpha^{r}\left[\mu_{f_{k}}(\alpha)\right]^{r / p} \frac{d \alpha}{\alpha}\right)^{p / r} \leq \sum_{k}\left\|f_{k}\right\|_{p, r}^{p}
\end{aligned}
$$

here we have used Minkowski's inequality in $L^{r / p}$ (and thus our assumption $r \geq p$ ).

We now further decompose $g_{k, \alpha}=l_{k, \alpha}+m_{k, \alpha}$ into a low and a middle part where for a suitable constant $B>1$,

$$
l_{k, \alpha}(x)= \begin{cases}f_{k}(x) & \text { if }\left|f_{k}(x)\right| \leq \alpha / B \\ 0 & \text { if }\left|f_{k}(x)\right|>\alpha / B\end{cases}
$$

and

$$
m_{k, \alpha}(x)= \begin{cases}0 & \text { if }\left|f_{k}(x)\right| \leq \alpha / B \\ f_{k}(x) & \text { if } \alpha / B<\left|f_{k}(x)\right| \leq \alpha \\ 0 & \text { if }\left|f_{k}(x)\right|>\alpha\end{cases}
$$

For a favorable choice for $B$ see (67) below. Now

$$
\begin{aligned}
& \mu\left(\left\{x: \sum_{k}\left|g_{k, \alpha}(x)\right|>\alpha\right\}\right) \\
& \quad \leq \mu\left(\left\{x: \sum_{k}\left|l_{k, \alpha}(x)\right|>\alpha / 2\right\}\right)+\mu\left(\left\{x: \sum_{k}\left|m_{k, \alpha}(x)\right|>\alpha / 2\right\}\right)
\end{aligned}
$$


and therefore by Minkowski's inequality and a subsequent change of variable

$$
\begin{aligned}
& \left(r \int_{0}^{\infty} \alpha^{r}\left[\mu\left(\left\{x: \sum_{k}\left|g_{k, \alpha}(x)\right|>\alpha\right\}\right)\right]^{r / p} \frac{d \alpha}{\alpha}\right)^{p / r} \\
& \leq 2^{p}\left(r \int_{0}^{\infty} \alpha^{r}\left[\mu\left(\left\{x: \sum_{k}\left|l_{k, 2 \alpha}(x)\right|>\alpha\right\}\right)\right]^{r / p} \frac{d \alpha}{\alpha}\right)^{p / r} \\
& +2^{p}\left(r \int_{0}^{\infty} \alpha^{r}\left[\mu\left(\left\{x: \sum_{k}\left|m_{k, 2 \alpha}(x)\right|>\alpha\right\}\right)\right]^{r / p} \frac{d \alpha}{\alpha}\right)^{p / r} .
\end{aligned}
$$

Next, by Tshebyshev's inequality

$$
\begin{aligned}
& \left(r \int_{0}^{\infty} \alpha^{r}\left[\mu\left(\left\{x: \sum_{k}\left|l_{k, 2 \alpha}(x)\right|>\alpha\right\}\right)\right]^{r / p} \frac{d \alpha}{\alpha}\right)^{p / r} \\
& \leq\left(r \int_{0}^{\infty} \alpha^{r}\left[\frac{1}{\alpha} \int \sum_{k}\left|l_{k, 2 \alpha}\right| d \mu\right]^{r / p} \frac{d \alpha}{\alpha}\right)^{p / r} \\
& \leq\left(r \int_{0}^{\infty}\left[\sum_{k} \alpha^{p} \frac{1}{\alpha} \int_{0}^{2 B^{-1} \alpha} \mu_{l_{k, 2 \alpha}}(\beta) d \beta\right]^{r / p} \frac{d \alpha}{\alpha}\right)^{p / r}
\end{aligned}
$$

here we have used $\mu_{l_{k, 2 \alpha}}(\beta)=0$ when $\beta>B^{-1} 2 \alpha$. We now use Minkowski's inequality in $L^{r / p}(d \alpha / \alpha)$. Since $\left|l_{k, 2 \alpha}\right| \leq\left|f_{k}\right|$ we see that the last displayed expression is dominated by

$$
\begin{aligned}
& \sum_{k}\left(r \int_{0}^{\infty}\left[\alpha^{p} \frac{1}{\alpha} \int_{0}^{2 B^{-1} \alpha} \mu_{f_{k}}(\beta) d \beta\right]^{r / p} \frac{d \alpha}{\alpha}\right)^{p / r} \\
& =\sum_{k}\left(r \int_{0}^{\infty} \alpha^{r}\left[\int_{0}^{2 B^{-1}} \mu_{f_{k}}(s \alpha) d s\right]^{r / p} \frac{d \alpha}{\alpha}\right)^{p / r} .
\end{aligned}
$$

We continue as in the proof of Hardy's inequalities and estimate using the integral Minkowski inequality

$$
\begin{aligned}
& \left(r \int_{0}^{\infty} \alpha^{r}\left[\int_{0}^{2 B^{-1}} \mu_{f_{k}}(s \alpha) d s\right]^{r / p} \frac{d \alpha}{\alpha}\right)^{p / r} \\
& \leq \int_{0}^{2 B^{-1}} s^{-p}\left(\int_{0}^{\infty} r \beta^{r-1} \mu_{f_{k}}(\beta)^{r / p} d \beta\right)^{p / r}=\frac{2^{1-p} B^{p-1}}{1-p}\left\|f_{k}\right\|_{L^{p, r}}^{p}
\end{aligned}
$$

Thus, combining estimates we get

$$
\text { (64a) } \leq \frac{2}{1-p} B^{p-1} \sum_{k}\left\|f_{k}\right\|_{p, r}^{p} \text {. }
$$

We now estimate the terms in (64b). We write $[0, \infty)$ as a union over the intervals $I_{n}=\left[B^{n}, B^{n+1}\right], n \in \mathbb{Z}$ and apply Lemma B.2 to each interval. 
Then (64b) is estimated by

$$
\begin{aligned}
& 2^{p}\left(\sum_{n \in \mathbb{Z}} r \int_{B^{n}}^{B^{n+1}} \alpha^{r}\left[\mu\left(\left\{x: \sum_{k}\left|m_{k, 2 \alpha}(x)\right|>\alpha\right\}\right)\right]^{r / p} \frac{d \alpha}{\alpha}\right)^{p / r} \\
& \leq 2^{p}\left(\sum_{n \in \mathbb{Z}}\left[p \int_{B^{n}}^{B^{n+1}} \alpha^{p} \mu\left(\left\{x: \sum_{k}\left|m_{k, 2 \alpha}(x)\right|>\alpha\right\}\right) \frac{d \alpha}{\alpha}\right]^{r / p}\right)^{p / r} .
\end{aligned}
$$

We now define

$$
f_{k, n}(x)= \begin{cases}f_{k}(x) & \text { if } B^{n} \leq\left|f_{k}(x)\right| \leq B^{n+2} \\ 0 & \text { otherwise }\end{cases}
$$

and observe

$$
\left|m_{k, 2 \alpha}(x)\right| \leq\left|f_{k, n}(x)\right| \text { if } B^{n} \leq \alpha \leq B^{n+1}
$$

Hence we get

$$
\begin{aligned}
& \left(r \int_{0}^{\infty} \alpha^{r}\left[\mu\left(\left\{x: \sum_{k}\left|m_{k, 2 \alpha}(x)\right|>\alpha\right\}\right)\right]^{r / p} \frac{d \alpha}{\alpha}\right)^{p / r} \\
& \leq\left(\sum_{n=-\infty}^{\infty}\left[\int_{0}^{\infty} p \alpha^{p-1} \mu\left(\left\{x: \sum_{k}\left|f_{k, n}(x)\right|>\alpha\right\}\right) d \alpha\right]^{r / p}\right)^{p / r} \\
& \leq\left(\sum_{n=-\infty}^{\infty}\left\|\sum_{k}\left|f_{k, n}\right|\right\|_{p}^{r}\right)^{p / r} \leq\left(\sum_{n=-\infty}^{\infty}\left[\sum_{k}\left\|f_{k, n}\right\|_{p}^{p}\right]^{r / p}\right)^{p / r} \\
& \leq \sum_{k}\left(\sum_{n=-\infty}^{\infty}\left\|f_{k, n}\right\|_{p}^{r}\right)^{p / r} .
\end{aligned}
$$

Here we have used the triangle inequality in $L^{p}, p<1$ and Minkowski's inequality for the sequence space $\ell^{r / p}, r \geq p$. Now

$$
\begin{aligned}
& \left\|f_{k, n}\right\|_{p}^{p} \leq \int_{0}^{B^{n}} p \alpha^{p-1} \mu_{f_{k}}(B) d \alpha+\int_{B^{n}}^{B^{n+2}} p \alpha^{p} \mu_{f_{k}}(\alpha) \frac{d \alpha}{\alpha} \\
& \leq B^{n p} \mu_{f_{k}}(B)+\left(\log B^{2}\right)^{1-p / r} \frac{p}{r^{p / r}}\left(\int_{B^{n}}^{B^{n+2}} r \alpha^{r} \mu_{f_{k}}(\alpha)^{r / p} \frac{d \alpha}{\alpha}\right)^{p / r}
\end{aligned}
$$

by Hölder's inequality, and

$$
\begin{aligned}
\left(\sum_{n}\left\|f_{k, n}\right\|_{p}^{r}\right)^{p / r} \leq & \left(\sum_{n} B^{n r} \mu_{f_{k}}(B)^{r / p}\right)^{p / r} \\
& +\left(\log B^{2}\right)^{1-p / r} \frac{p}{r^{p / r}}\left(\sum_{n} \int_{B^{n}}^{B^{n+2}} r \alpha^{r-1} \mu_{f_{k}}(\alpha)^{r / p} d \alpha\right)^{r / p} \\
\leq & \left(1+\frac{p}{r^{p / r}} 2^{p / r}\left(\log B^{2}\right)^{1-p / r}\right)\left(\int_{0}^{\infty} r \alpha^{r-1} \mu_{f_{k}}(\alpha) d \alpha\right)^{p / r}
\end{aligned}
$$




\section{Consequently}

$$
(\underline{64 \mathrm{~b}}) \leq 2^{p}\left(1+\frac{p}{r^{p / r}} 2^{p / r}(2 \log B)^{1-p / r}\right) \sum_{k}\left\|f_{k}\right\|_{L^{p, r}}^{p} .
$$

We now make the choice

$$
B=(1-p)^{-\frac{p}{r(1-p)}}
$$

so that

$$
\frac{B^{p-1}}{1-p}=(1-p)^{-(1-p / r)}, \quad \log B=\frac{p}{r(1-p)} \log \frac{1}{p-1}
$$

Combining the various estimates we obtain

$$
\begin{aligned}
& \left\|\sum_{k} f_{k}\right\|_{L^{p, r}}^{p} \\
& \leq\left(1+\frac{2}{(1-p)^{1-p / r}}+\frac{p 2^{p+1}}{r^{p / r}} \frac{p}{r(1-p)} \log \left(\frac{1}{1-p}\right)^{1-p / r}\right) \sum_{k}\left\|f_{k}\right\|_{L^{p, r}}^{p}
\end{aligned}
$$

which yields the lemma.

\section{REFERENCES}

[1] Albert Baernstein, II; Eric T. Sawyer. Embedding and multiplier theorems for $H^{p}\left(\mathbb{R}^{n}\right)$. Mem. Amer. Math. Soc. 53 (1985), no. 318.

[2] Sorina Barza, Viktor Kolyada, Javier Soria. Sharp constants to the triangle inequality in Lorentz spaces. Trans. Amer. Math. Soc. 361, no. 10 (2009).

[3] Colin Bennett and Robert Sharpley. Interpolation of operators, Academic Press, 1988.

[4] Joram Bergh and Jörgen Löfström. Interpolation Spaces. An Introduction. Springer, Berlin, 1976.

[5] Andrea Cianchi, Luboš Pick. An optimal endpoint trace embedding. Ann. Inst. Fourier (Grenoble) 60 (2010), no. 3, 939-951.

[6] Charles Fefferman, Elias M. Stein. Some maximal inequalities. Amer. J. Math. 93 (1971) 107-115.

[7] Jens Franke. On the spaces $F_{p q}^{s}$ of Triebel-Lizorkin type: pointwise multipliers and spaces on domains. Math. Nachr. 125 (1986), 29- 68.

[8] Amiran Gogatishvili, Bohumír Opic, Sergey Tikhonov, Walter Trebels. Ulyanov-type inequalities between Lorentz-Zygmund spaces. J. Fourier Anal. Appl. 20 (2014), no. $5,1020-1049$.

[9] Loukas Grafakos, Lenka Slavíková. A sharp version of the Hörmander multiplier theorem. To appear in International Mathematics Research Notices.

[10] Lars Inge Hedberg, Yuri Netrusov. An axiomatic approach to function spaces, spectral synthesis, and Luzin approximation. Mem. Amer. Math. Soc. 188 (2007), no. 882, vi+97 pp.

[11] Lars Hörmander. Estimates for translation invariant operators in $L^{p}$ spaces. Acta Math. 104 (1960), 93-140.

[12] Richard Hunt. On $L(p, q)$ spaces. Enseignement Math. (2) 12 (1966), 249-276.

[13] Björn Jawerth. Some observations on Besov and Lizorkin-Triebel spaces, Math. Scand. 40 (1977), no. 1, 94-104.

[14] Nigel J. Kalton. Linear operators on $L^{p}$ for $0<p<1$. Trans. Amer. Math. Soc. 259 (1980), no. 2, 319-355. 
[15] Hyerim Ko, Sanghyuk Lee. Fourier transform and regularity of characteristic functions. Proc. Amer. Math. Soc. 145 (2017), no. 3, 1097-1107.

[16] G.G. Lorentz. On the theory of spaces $\Lambda$. Pacific J. Math. 1, (1951), 411-429.

[17] Jaak Peetre. On spaces of Triebel-Lizorkin type. Ark. Mat. 13 (1975), 123-130.

[18] Andreas Seeger. A limit case of the Hörmander multiplier theorem. Monatsh. Math. 105 (1988), 151-160.

[19] Estimates near $L^{1}$ for Fourier multipliers and maximal functions. Arch. Math. (Basel) 53 (1989), 188-193.

[20] Remarks on singular convolution operators. Studia Math. 97 (1990), no. 2, 91-114.

[21] Winfried Sickel and Hans Triebel. Hölder inequalities and sharp embeddings in function spaces of $B_{p q}^{s}$ and $F_{p q}^{s}$ type, Z. Anal. Anwendungen 14 (1995), no. 1, 105-140.

[22] Lenka Slavíková. On the failure of the Hörmander multiplier theorem in a limiting case. Preprint, May 2018, arXiv:1805.09907.

[23] Elias M. Stein. Singular integrals and differentiability of functions. Princeton University Press, Princeton, New Jersey, 1970.

[24] Editor's note: the differentiability of functions in $\mathbb{R}^{n}$. Ann. of Math. (2) 113 (1981), no. 2, 383-385.

[25] Elias M. Stein, Mitchell H. Taibleson, Guido Weiss. Weak type estimates for maximal operators on certain $H^{p}$ classes. Proceedings of the Seminar on Harmonic Analysis (Pisa, 1980). Rend. Circ. Mat. Palermo (2) 1981, suppl. 1, 81-97.

[26] Elias M. Stein, Guido Weiss. Introduction to Fourier analysis on Euclidean spaces. Princeton Mathematical Series, No. 32. Princeton University Press, Princeton, N.J., 1971.

[27] Elias M. Stein, Norman J. Weiss. On the convergence of Poisson integrals. Trans. Amer. Math. Soc. 140 (1969), 35-54.

[28] Mitchell H. Taibleson. On the theory of Lipschitz spaces of distributions on Euclidean $n$-space. I. Principal properties. J. Math. Mech. 13 (1964), 407-479. On the theory of Lipschitz spaces of distributions on Euclidean $n$-space. II. Translation invariant operators, duality, and interpolation. J. Math. Mech. 14 (1965), 821-839.

[29] Hans Triebel. Theory of function spaces. Monographs in Mathematics, 78. Birkhäuser Verlag, Basel, 1983.

[30] . Theory of function spaces. II. Monographs in Mathematics, 84. Birkhäuser Verlag, Basel, 1992.

[31] - Tempered homogeneous function spaces. EMS Series of Lectures in Mathematics. European Mathematical Society (EMS), Zürich, 2015.

[32] Jan Vybíral. A new proof of the Jawerth-Franke embedding. Rev. Mat. Complut. 21 (2008), no. 1, 75-82.

Andreas Seeger, Department of Mathematics, University of Wisconsin, 480 Lincoln Drive, Madison, WI, 53706, USA

E-mail address: seeger@math.wisc.edu

Walter Trebels, Fachbereich Mathematik, Technische Universität DarmStadt, Schlossgartenstr. 7, 64289 Darmstadt, Germany

E-mail address: trebels@mathematik.tu-darmstadt.de 